\title{
Comparative efficacy and safety of antipsychotics in the treatment of schizophrenia: a network meta-analysis in a Japanese population
}

This article was published in the following Dove Press journal:

Neuropsychiatric Disease and Treatment

II May 2017

Number of times this article has been viewed

\author{
Taro Kishil,* \\ Toshikazu Ikuta ${ }^{2, *}$ \\ Shinji Matsunaga' \\ Yuki Matsuda ${ }^{1,3}$ \\ Kazuto Oya' \\ Nakao Iwata' \\ 'Department of Psychiatry, Fujita \\ Health University School of Medicine, \\ Toyoake, Aichi, Japan; '2Department \\ of Communication Sciences and \\ Disorders, School of Applied Sciences, \\ University of Mississippi, Oxford, \\ MS, USA; ${ }^{3}$ Department of Psychiatry, \\ National Center Hospital, National \\ Center of Neurology and Psychiatry, \\ Kodaira, Tokyo, Japan \\ *These authors contributed equally \\ to this work
}

Background: The relative efficacy and tolerability of antipsychotics for schizophrenia are considerably well studied. This study aimed to examine whether previous findings could be replicated in a genetically distinct and homogenous group (ie, Japanese patients with schizophrenia) and whether previous findings could be extended to a broader range of antipsychotics with previously unclear relative efficacy and tolerability.

Methods: Bayesian network meta-analysis was performed in which randomized trials comparing any of the following interventions were included: second-generation antipsychotics, haloperidol, or placebo. The primary outcomes for efficacy and acceptability were the response rate and all-cause discontinuation. The secondary outcomes included the improvement of Positive and Negative Syndrome Scale scores, discontinuation because of adverse events, and individual adverse events.

Results: Eighteen relevant studies were identified (total $n=3,446$; aripiprazole $=267$, blonanserin $=285$, clozapine $=47$, clocapramine $=295$, haloperidol $=857$, mosapramine $=493$, olanzapine $=179$, paliperidone $=136$, perospirone $=146$, placebo $=138$, quetiapine $=212$, and risperidone $=338$; mean study duration $=8.33 \pm 1.41$ weeks . In primary outcomes, olanzapine and paliperidone showed efficacy than placebo, and olanzapine and paliperidone showed superior acceptability compared with placebo. There were differences in the incidences of individual adverse events (the best antipsychotic: extrapyramidal symptoms = olanzapine, hyperprolactinemiarelated symptoms $=$ quetiapine, sedation $=$ paliperidone, and weight change $=$ blonanserin $)$ among antipsychotics.

Conclusion: Although the current analysis exclusively included Japanese patients with schizophrenia, no remarkable differences were observed in efficacy and safety compared with previous meta-analyses. Diverse hierarchies in safety outcomes also support the implication that individual risk expectations for adverse events can guide clinical decisions. However, the sample size was relatively limited. Additional efficacy and safety data are required to fully obtain a conclusive understanding.

Keywords: antipsychotics, Japanese schizophrenia, network meta-analysis, efficacy, safety

\section{Introduction}

A recent network meta-analysis provided evidence that there are considerable differences in the efficacy and risks of adverse events associated with 15 antipsychotics (clozapine, amisulpride, olanzapine, risperidone, paliperidone, zotepine, haloperidol, quetiapine, aripiprazole, sertindole, ziprasidone, chlorpromazine, asenapine, lurasidone, and iloperidone). ${ }^{1}$ Although clozapine demonstrated a superior overall change in symptoms over placebo with large effect size (standardized mean difference 
[SMD] $=-0.88)$, other antipsychotics showed only smallto-medium effect size (SMD $=-0.33$ to -0.66 ). Clozapine, zotepine, and olanzapine were associated with an increased weight gain risk, compared with placebo, with a large effect size ( $\mathrm{SMD}=-0.65$ to -0.74 ), and haloperidol, ziprasidone, and lurasidone were not associated with weight gain risk. A network meta-analysis can provide comprehensive and uniform information, aiding clinicians in evidence-based decision making.

Although the advantages of network meta-analyses are firmly established, previous studies did not wholly include antipsychotics that are recently entering the market, for example, blonanserin, clocapramine, mosapramine, and perospirone, which are classified as second-generation antipsychotics (SGAs) and are currently approved for schizophrenia treatment only in East Asia. It remains unclear where these newer drugs are placed in comparison with more widely marketed antipsychotics.

In addition to newer antipsychotics, factors of race/ ethnicity have not been accommodated in previous network meta-analyses of antipsychotics. A recent meta-analysis demonstrated that the effect size for the response to SGAs was smaller in patients with schizophrenia in North America than in those worldwide..$^{2-4}$ For the differences in incidence and/or magnitude of antipsychotic-induced adverse events among each race and ethnicity, olanzapine caused greater weight gain in African-American patients with schizophrenia than in Caucasian patients. ${ }^{5}$ In those without psychiatric disorders, there are inter-race differences in the prevalence of metabolic syndrome and predisposition factors (eg, diabetes mellitus). ${ }^{6,7}$ Although there is a lower prevalence of obesity in Japanese compared with other ethnic groups, there is a higher rate of diabetes mellitus. ${ }^{8}$ Metabolic syndrome, which results in obesity, is considered a complex disease, where insulin resistance pathophysiology likely involves a geneenvironment interaction. ${ }^{9,10}$

Genetic diversity at least partially accounts for these ethnic-dependent differences in disease prevalence. Genetic variations may mediate differences in the antipsychotic response and side effect development. ${ }^{11-13}$ The polymorphisms in cytochrome $\mathrm{P} 450 ;{ }^{14}$ the main metabolizer of antipsychotics) were different in each population. A recent genome-wide association study demonstrated that rs 489693 , located $\sim 190 \mathrm{~kb}$ downstream of the melanocortin 4 receptor gene, was associated with antipsychotic-induced weight gain. ${ }^{15}$ The minor allelic frequency of rs 489693 also differs between different races. ${ }^{14}$ Asian populations have been reported to exhibit a higher rate of agranulocytosis induced by clozapine than that in other populations. ${ }^{16}$ rs 1800625 in the human leukocyte antigen region was associated with clozapine-induced agranulocytosis and granulocytopenia in a Japanese population. ${ }^{17}$ The minor allelic frequency of rs1800625 also differs between various races. ${ }^{18}$ Thus, when data from various races or ethnicities are combined, the biological and environmental factors can notably influence the evidence regarding the efficacy and safety of antipsychotics.

Japanese individuals have a higher homogeneity of genetic and cultural backgrounds than other populations. ${ }^{19}$ The universal health care insurance system in Japan allows its population to obtain health services without suffering any financial hardship, reducing the selection bias toward the enrollment in clinical trials. Examining the replicability of the previous study in a genetically and physiologically more homogeneous sample can provide more conclusive understanding of the relative antipsychotic efficacy and tolerability. A Bayesian network meta-analysis was carried out to assess the relative efficacy and tolerability of SGAs (eg, aripiprazole, blonanserin, clozapine, clocapramine, mosapramine, olanzapine, paliperidone, perospirone, quetiapine, and risperidone) exclusively in Japanese patients with schizophrenia, to examine the replicability of previous findings for additional antipsychotics in a different population. The network meta-analysis has the following benefits compared with traditional meta-analyses: ${ }^{20} 1$ ) it increases statistical power by incorporating evidence from both direct and indirect comparisons across all interventions; 2) it combines direct and indirect evidence for any given pair of treatments in one joint analysis. In addition to analysis of the direct within-trial comparisons between two treatments, the network framework enabled the incorporation of indirect comparisons constructed from two trials that have one treatment in common.

\section{Methods}

This meta-analysis was performed according to the Preferred Reporting Items for Systematic Reviews and Meta-Analyses guidelines. ${ }^{21}$ The review has been registered with PROSPERO.22

\section{Inclusion criteria, search strategy, data extraction, and outcomes}

To conduct a Bayesian network meta-analysis, rater-blind or double-blind, randomized trials were first selected comparing any of the following interventions: SGAs (eg, aripiprazole, blonanserin, clozapine, clocapramine, mosapramine, olanzapine, paliperidone, perospirone, quetiapine, or risperidone) approved for treating schizophrenia in Japan, as 
well as haloperidol, chlorpromazine, or placebo. Open-label and crossover studies were excluded to avoid any potential bias, such as performance, detection bias, and the carry over effect. PubMed, ${ }^{23}$ databases of the Cochrane Library, ${ }^{24}$ and the Japan Medical Abstracts Society ${ }^{25}$ were searched, as well as the reference lists of relevant articles for trials published between January 1, 1970, and November 10, 2016. The following keywords were used: "Japan" or "Japanese" AND "aripiprazole," "blonanserin," "clozapine," "chlorpromazine," "clocapramine," "mosapramine," "olanzapine," "paliperidone," "perospirone," "quetiapine," "risperidone," "haloperidol," or "placebo" AND "schizophrenia" in English and Japanese. In addition, the drug package insert for each antipsychotic ${ }^{26}$ was also used. Inquiries were made with pharmaceutical companies regarding various clinical trials. Three authors (TK, YM, and SM) checked the inclusion and exclusion criteria of the studies that were identified. When the data required for the current metaanalysis were missing, the first or corresponding authors were contacted for additional information. Unpublished data were provided for the current study by Dr Kotaro Hatta, Otsuka Pharmaceutical Co Ltd (Tokyo, Japan), Eli Lilly and Company (Indianapolis, IN, USA), Janssen Pharmaceutical KK (Tokyo, Japan), Astellas Pharma Inc. (Tokyo, Japan), AstraZeneca plc (London, UK), and Dainippon Sumitomo Pharma (Osaka, Japan). Discrepancies in the two coding forms were resolved by discussions between two authors (TK and NI). TK, SM, YM, and KO independently extracted and checked the data.

\section{Data synthesis and statistical analysis}

The primary outcomes for efficacy and acceptability were response rate (Supplementary material 1) and all-cause discontinuation. The secondary outcomes included the improvement of the Positive and Negative Syndrome Scale (PANSS $)^{27}$ total; the positive, negative, and general subscale scores; discontinuation because of adverse events; and individual adverse events. A modified intention-to-treat population was used in the analysis, which is also known as full analysis set (FAS; ie, at least one dose or at least one follow-up assessment) data or per-protocol population (PPP; ie, individuals who perfectly adhered to eligibility, interventions, and outcome assessment) data (Table 1).

A pairwise meta-analysis and Bayesian network metaanalysis with a random effects model ${ }^{28}$ were performed by using the GeMTC package in R software.$^{29}$ Because random effects models are more conservative than a fixed effect model, ${ }^{30}$ the random effects model was used for all metaanalyses. Direct and indirect evidence from all relevant studies

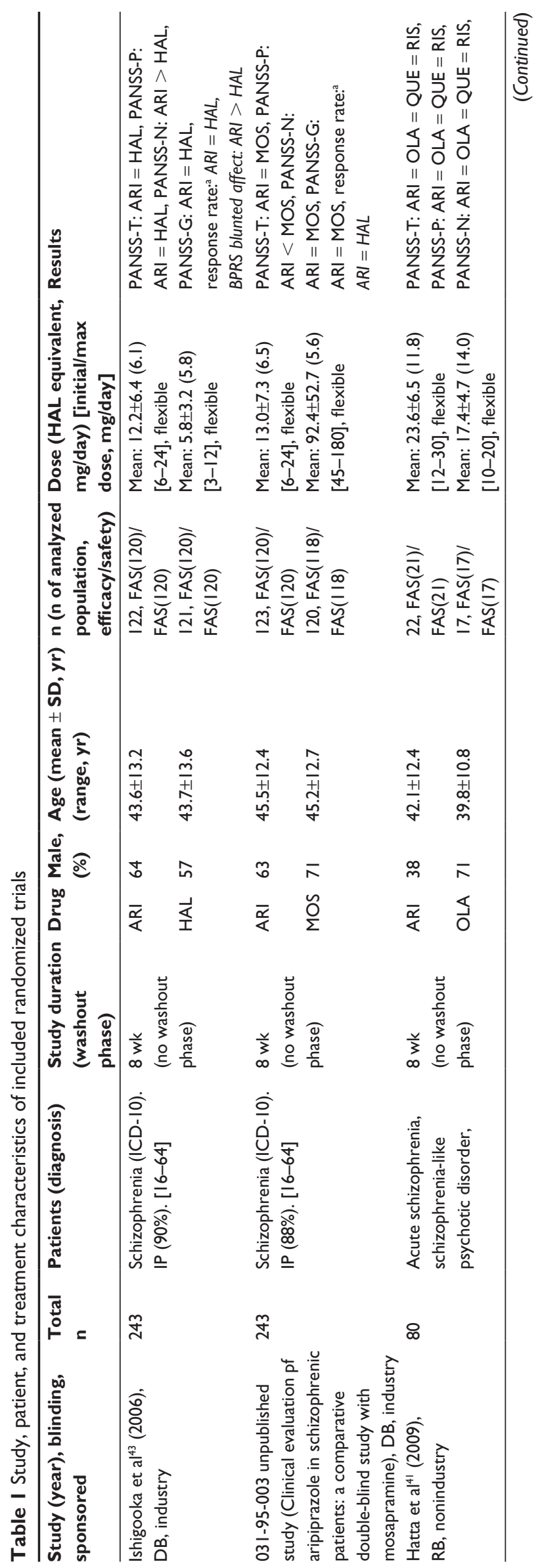




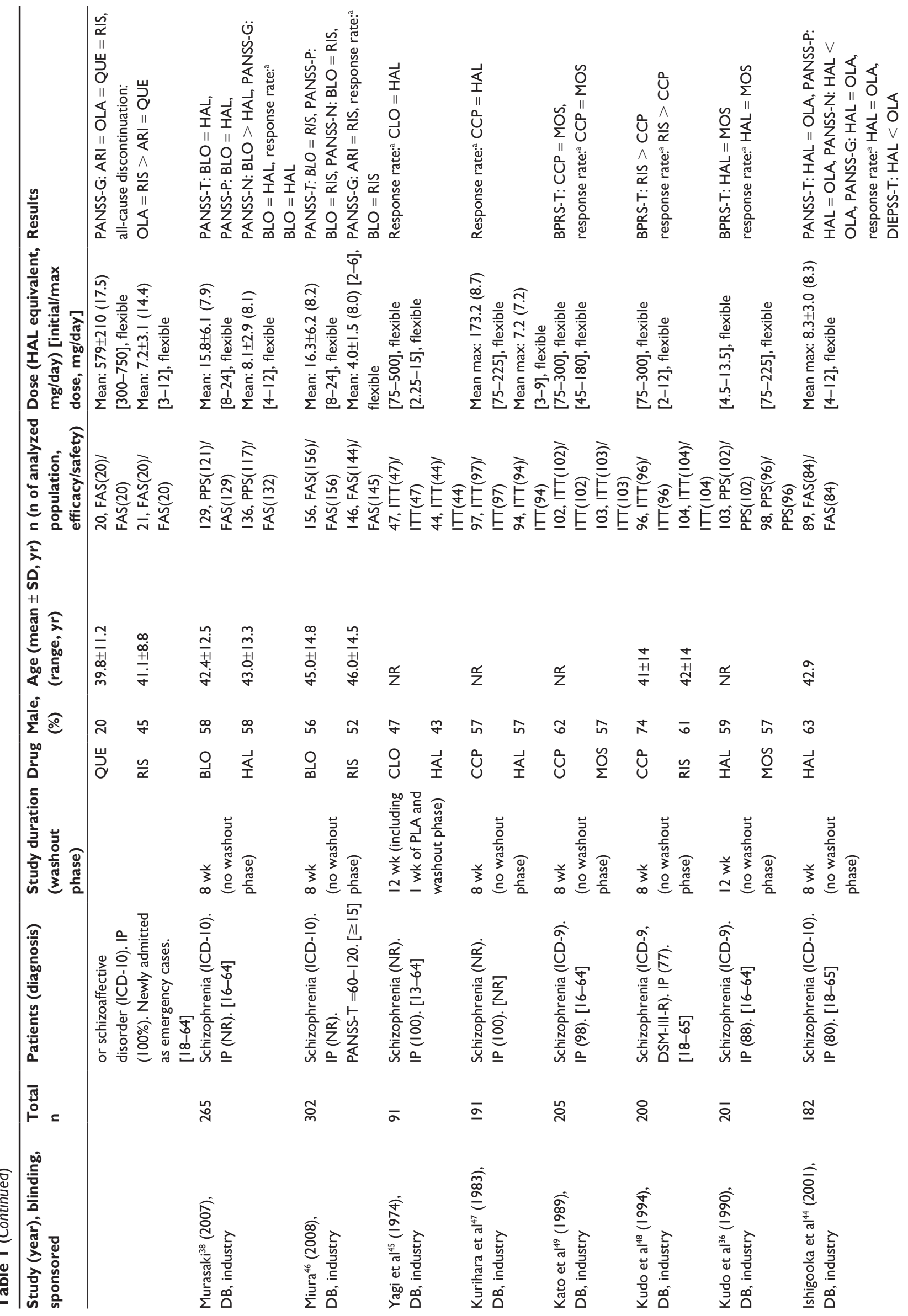




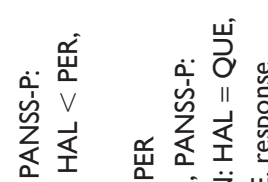

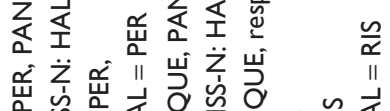

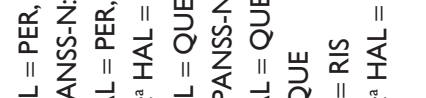

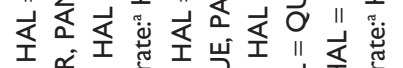

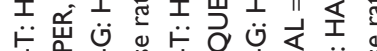

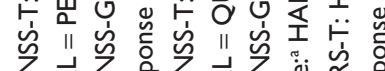

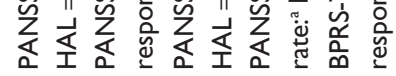

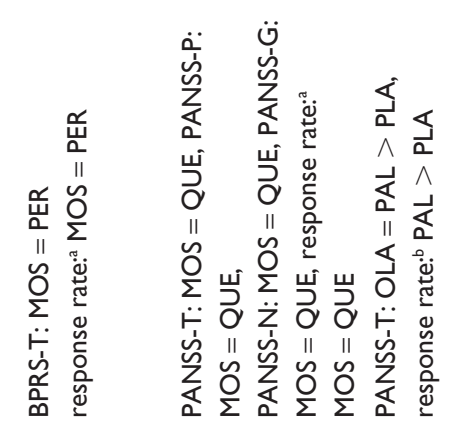

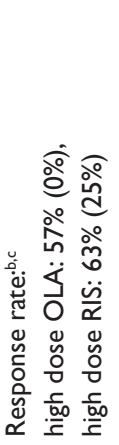

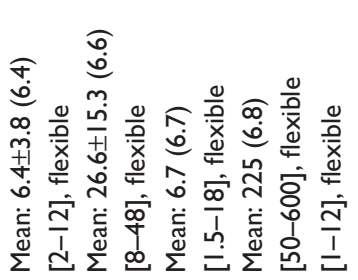

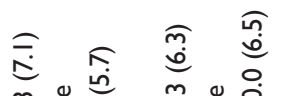

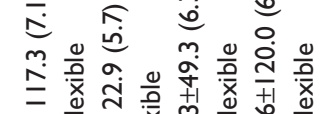

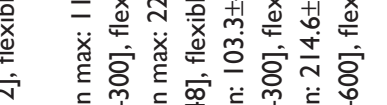

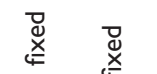

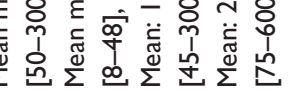

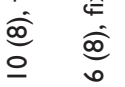

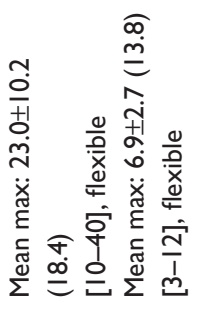

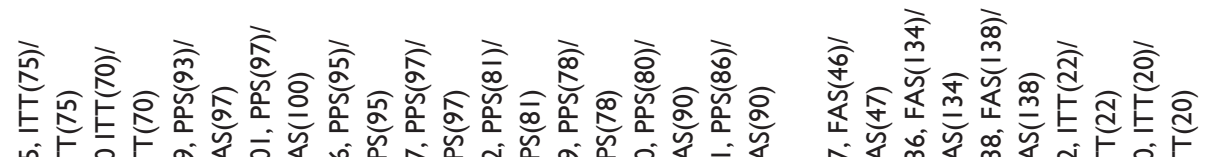

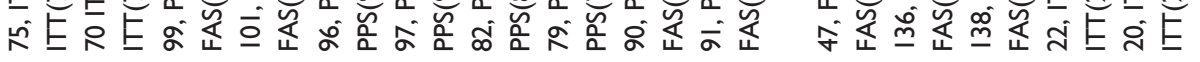

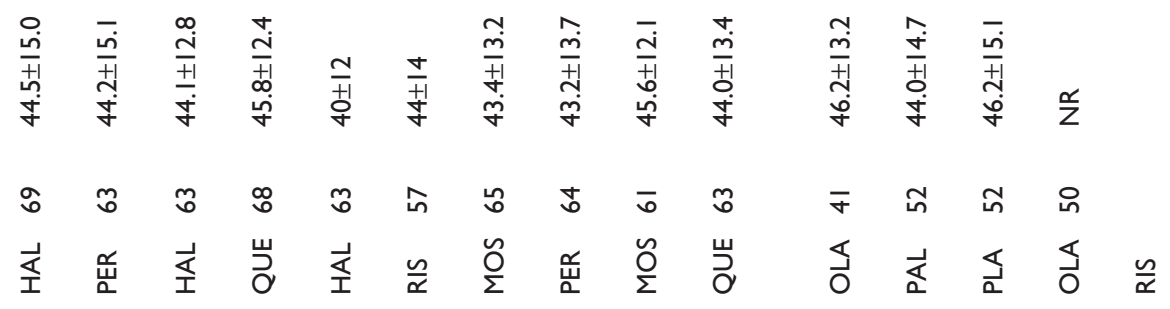

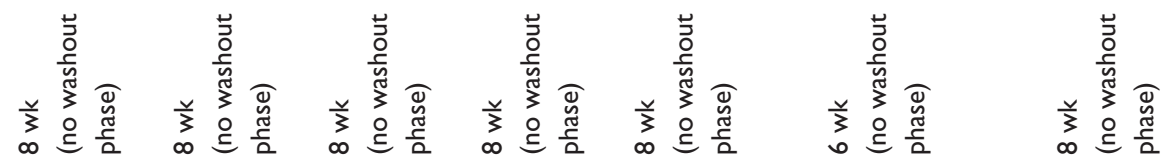

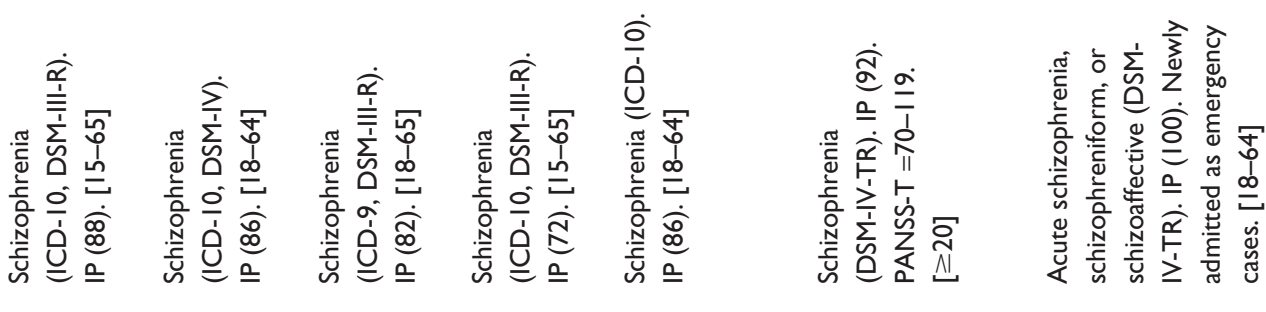

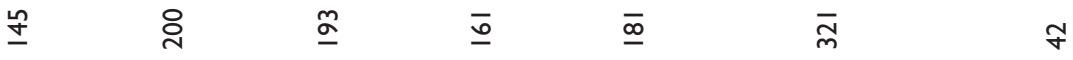

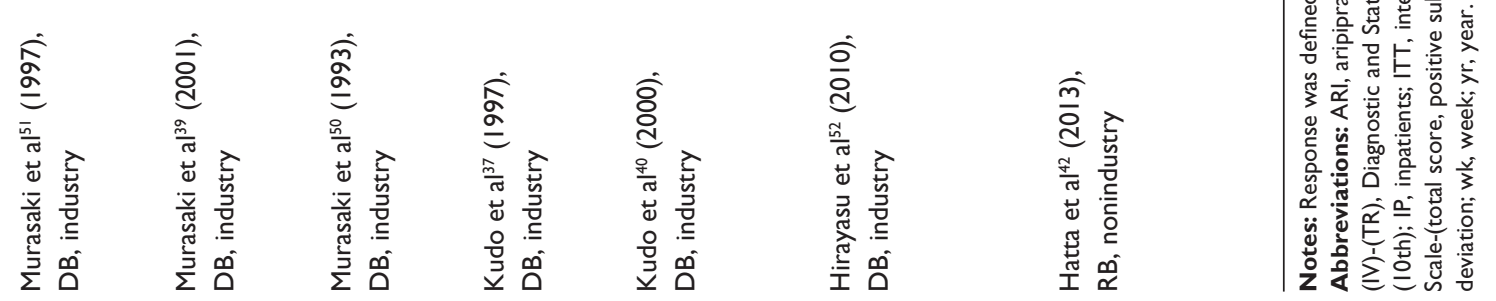


was integrated by using a Bayesian network meta-analysis, and estimates of the maximum power were provided. ${ }^{31}$ Mean differences (MDs) and the log odds ratio (LOR) were used for continuous and dichotomous data, respectively, along with its $95 \%$ credible intervals (the Bayesian analogs of $95 \%$ confidence intervals [CIs]). Bayesian analyses were performed by using a Markov chain Monte Carlo simulation to calculate the MDs, LORs, and CIs. ${ }^{29}$ The simulation generated 200,000 iterations, and the convergence was assessed by using the Brooks-Gelman-Rubin diagnostic. ${ }^{32}$ GeMTC can set the prior distributions automatically; the most straightforward way to ensure their sufficient vagueness is to set the outcome scale parameter. Model selection (consistency model, unrelated mean effects model, or unrelated study effects model) was based on the Dias guidelines for evaluating linear models. ${ }^{33,34}$ A lower deviance information criterion (DIC) value indicates a better model fit. Heterogeneity between trial results was tested with an $I^{2}$ statistic (considering values $\geq 50 \%$ to reflect considerable heterogeneity ${ }^{35}$ ). The potential for inconsistency was assessed by an inspection of the network plots. Where there was a potential for inconsistency (ie, where there were independent sources of evidence informing direct and indirect estimates), Bayesian $P$-values for the difference between direct and indirect evidence were calculated by using the node split method, ${ }^{33,34}$ implemented in R through GeMTC, ${ }^{29}$ and direct and indirect estimates were compared $(P<0.05$ was set as a significant inconsistency). Using the Bayesian approach, the relative effect estimates were calculated and could be used to estimate the probability that one was superior to the other. In the Markov chain Monte Carlo cycle, each ventilation strategy is ranked based on the estimated effect size. These probabilities total a value of 1 for each treatment and rank.

\section{Results}

\section{Study characteristics}

Of the 6,469 hits, 4,485 duplicates, 1,955 references based on an abstract/title review, and 26 articles after a full-text review (21 review articles, 3 open-label studies, and 2 crossover studies) were removed, leaving 3 remaining studies for the systematic review (Supplementary material 2). Moreover, 15 additional studies were retrieved by searching through the review articles and the drug package inserts, for a total of 18 studies (Supplementary material 2). Eighteen relevant studies were identified (total $n=3,446$; aripiprazole $=267$, blonanserin $=285$, clozapine $=47$, clocapramine $=295$, haloperidol $=857$, mosapramine $=493$, olanzapine $=179$, paliperidone $=136$, perospirone $=146$, placebo $=138$, quetiapine $=212$, and risperidone $=338) .{ }^{36-52}$ One of these trials was a four-arm experiment, ${ }^{41}$ another one was a three-arm experiment, ${ }^{52}$ and the remaining trials were two-arm experiments. Only an olanzapine versus risperidone comparison included plural studies (there were no significant heterogeneities in all outcomes, including data from plural olanzapine vs risperidone comparisons; data not shown). ${ }^{41,42}$ One of these trials was a placebocontrolled study. ${ }^{52}$ Two studies ${ }^{41,42}$ were rater-blinded and nonindustry-sponsored studies, and the remaining trials were double-blinded and industry-sponsored studies. One of these trials was an unpublished study (031-95-003; Clinical evaluation of aripiprazole in schizophrenic patients: a comparative double-blind study with mosapramine). Although three of the studies used FAS for efficacy outcomes, all studies used FAS for safety outcomes. Three ${ }^{41,42,44}$ of the studies were published in English. Because most of all Japanese trials were published in Japanese, they did not hit in the literature search using PubMed and databases of the Cochrane Library. The mean study duration was $8.33 \pm 1.41$ weeks (range: 6-12 weeks), with sample sizes of 17-156 participants per treatment arm. All participants were adults diagnosed with schizophrenia, and the mean age was 47 years. The proportion of males was 59.2\%. Patients in all studies included in the meta-analysis were adults (there were no studies that included specifically adolescent or elderly patients). Two studies $^{41,42}$ only included emergency cases. In 15 studies, there was at least one patient who used antipsychotics other than the intervention as concomitant drugs at least once during the study (mean: $9.1 \%$; range: $0.1 \%-30.4 \%$ ). In 16 studies, there was at least one patient who used benzodiazepine as a concomitant drug at least once during the study (mean: $25.8 \%$; range: $1.0 \%-95.2 \%$ ). The characteristics of the studies are listed in Table 1.

\section{Results of the network meta-analysis}

No significant heterogeneity in the primary outcomes was found between the antipsychotics for a pairwise meta-analysis (only olanzapine vs risperidone: data not shown). The Supplementary materials 3-5 show the network of eligible comparisons (Supplementary material 3), the selection of the goodness-of-fit for the model (Supplementary $\underline{\text { material } 4)}$, and the results of the node-splitting inconsistency model (Supplementary material 5). The consistency model showed the lowest DIC value with respect to all outcomes (Supplementary material 4). The results of the network meta-analysis and rank probabilities with respect to all outcomes are included in Supplementary material 6. 
The findings from the network meta-analyses can be summarized as follows: 1) in response rate, olanzapine and paliperidone were more effective (response rate) compared with placebo; 2) olanzapine and paliperidone showed greater acceptability (all-cause discontinuation) than placebo; and 3) diverse characteristics were observed in adverse events among antipsychotics. Results from each of the outcomes are as follows.

\section{Efficacy outcomes}

The simple pairwise meta-analyses did not reveal any significant differences between any of the antipsychotics for any of the efficacy outcomes. The results of the network meta-analyses are described below.

\section{Response rate (primary outcome for efficacy)}

Olanzapine and paliperidone demonstrated significant superiority to placebo, whereas the other antipsychotics failed to show any differences from placebo. Although not significantly superior to placebo, the most effective antipsychotic was clozapine, followed by quetiapine. A significant disagreement between the direct and indirect estimates (hereafter inconsistency) was detected between risperidone and haloperidol (Table 2).

\section{PANSS total scores}

Olanzapine and paliperidone were significantly superior to placebo. Olanzapine was ranked the highest in improving PANSS total scores; paliperidone was the second-ranked antipsychotic. No inconsistency was detected (Table 3).

\section{PANSS positive subscale scores}

Olanzapine and paliperidone were superior to placebo. Paliperidone was ranked the highest in improving PANSS positive subscale scores, and olanzapine was ranked second. No inconsistency was detected (Table 3).

\section{PANSS negative subscale scores}

Olanzapine was superior to placebo. Although not significantly superior to placebo, perospirone improved PANSS negative subscale scores the most, and olanzapine was the second-ranked antipsychotic. No inconsistency was detected (Table 4).

\section{PANSS general subscale scores}

Olanzapine and paliperidone were superior to placebo. Olanzapine exhibited the highest improving PANSS general subscale scores. Mosapramine was the second-ranked antipsychotic. No inconsistency was detected (Table 4).

\section{Acceptability/safety outcomes}

All-cause discontinuation (primary outcome for acceptability)

Olanzapine and risperidone were superior to quetiapine in the pairwise meta-analysis. Olanzapine was also superior to aripiprazole. However, no significant differences were found among other comparisons. The inconsistency was detected only regarding olanzapine versus quetiapine. In the network meta-analysis, olanzapine was superior to aripiprazole, haloperidol, mosapramine, and placebo. Paliperidone was superior to placebo. Risperidone was superior to haloperidol. Paliperidone was the highest ranked antipsychotic with respect to all-cause discontinuation; olanzapine was the second-ranked antipsychotic, followed by risperidone (Table 2 ).

\section{Discontinuation due to adverse events}

Olanzapine was superior to aripiprazole in the pairwise metaanalysis, and clocapramine was superior to mosapramine. No inconsistency was found between any of the antipsychotics. In the network meta-analysis, olanzapine was superior to aripiprazole, haloperidol, and mosapramine. Paliperidone was associated with the lowest risk of this outcome, and olanzapine was ranked second (Table 5).

\section{At least one adverse event}

Aripiprazole was superior to haloperidol in the pairwise meta-analysis. The network meta-analysis revealed that aripiprazole and quetiapine were superior to clocapramine, haloperidol, and mosapramine. Aripiprazole was associated with the lowest risk of this outcome, and quetiapine was the second-ranked antipsychotic. No inconsistencies were detected (Table 5).

\section{Agitation, anxiety, and the use of anxiolytics}

Quetiapine was superior to aripiprazole and risperidone in the pairwise meta-analysis. The network meta-analysis did not show any significant differences between any of the antipsychotics. Clozapine was associated with the lowest risk of this outcome, and quetiapine was the second-ranked antipsychotic. Inconsistencies were found between aripiprazole and quetiapine, as well as between quetiapine and risperidone (Table 6).

\section{Insomnia and the use of hypnotics}

Olanzapine was superior to haloperidol in the pairwise meta-analysis. In the network meta-analysis, clozapine 
Table 2 Network meta-analysis of the primary outcomes regarding efficacy (response rate) and acceptability (all-cause discontinuation)

\begin{tabular}{|c|c|c|c|c|c|}
\hline ARI & $\begin{array}{l}-0.3709 \\
(-1.187,0.3909)\end{array}$ & $\begin{array}{l}-0.3614 \\
(-2.035,1.256)\end{array}$ & $\begin{array}{l}-0.5582 \\
(-1.371,0.2225)\end{array}$ & $\begin{array}{l}-0.01501 \\
(-0.5857,0.52)\end{array}$ & $\begin{array}{l}-0.1117 \\
(-0.6727,0.4586)\end{array}$ \\
\hline $\begin{array}{l}-0.04782 \\
(-0.7893,0.8506)\end{array}$ & BLO & $\begin{array}{l}0.01577 \\
(-1.671,1.672)\end{array}$ & $\begin{array}{l}-0.1884 \\
(-1.001,0.6472)\end{array}$ & $\begin{array}{l}0.3551 \\
(-0.2359,0.9716)\end{array}$ & $\begin{array}{l}0.2589 \\
(-0.4734,1.053)\end{array}$ \\
\hline $\begin{array}{l}0.3916 \\
(-0.9345,1.793)\end{array}$ & $\begin{array}{l}0.4267 \\
(-0.9455,1.804)\end{array}$ & CLO & $\begin{array}{l}-0.1986 \\
(-1.894,1.508)\end{array}$ & $\begin{array}{l}0.3428 \\
(-1.206,1.918)\end{array}$ & $\begin{array}{l}0.2502 \\
(-1.359,1.917)\end{array}$ \\
\hline $\begin{array}{l}-0.3738 \\
(-1.072,0.4409)\end{array}$ & $\begin{array}{l}-0.3277 \\
(-1.12,0.4316)\end{array}$ & $\begin{array}{l}-0.7599 \\
(-2.14,0.5976)\end{array}$ & Cloca & $\begin{array}{l}0.5449 \\
(-0.1212,1.21)\end{array}$ & $\begin{array}{l}0.4498 \\
(-0.2379,1.175)\end{array}$ \\
\hline $\begin{array}{l}-0.2425 \\
(-0.7613,0.392)\end{array}$ & $\begin{array}{l}-0.1969 \\
(-0.8308,0.4037)\end{array}$ & $\begin{array}{l}-0.6279 \\
(-1.875,0.5884)\end{array}$ & $\begin{array}{l}0.1309 \\
(-0.4539,0.7112)\end{array}$ & HAL & $\begin{array}{l}-0.09479 \\
(-0.5797,0.4242)\end{array}$ \\
\hline $\begin{array}{l}-0.05562 \\
(-0.6108,0.5864)\end{array}$ & $\begin{array}{l}-0.007263 \\
(-0.8086,0.7251)\end{array}$ & $\begin{array}{l}-0.4409 \\
(-1.79,0.8777)\end{array}$ & $\begin{array}{l}0.3208 \\
(-0.3276,0.9289)\end{array}$ & $\begin{array}{l}0.1905 \\
(-0.3266,0.6729)\end{array}$ & MOS \\
\hline $\begin{array}{l}0.1769 \\
(-0.5983,1.129)\end{array}$ & $\begin{array}{l}0.2191 \\
(-0.6325,1.143)\end{array}$ & $\begin{array}{l}-0.2038 \\
(-1.602,1.214)\end{array}$ & $\begin{array}{l}0.5515 \\
(-0.2748,1.452)\end{array}$ & $\begin{array}{l}0.421 \\
(-0.2317,1.162)\end{array}$ & $\begin{array}{l}0.2326 \\
(-0.5323,1.107)\end{array}$ \\
\hline $\begin{array}{l}0.1594 \\
(-1.071,1.565)\end{array}$ & $\begin{array}{l}0.2005 \\
(-1.095,1.578)\end{array}$ & $\begin{array}{l}-0.2258 \\
(-1.935,1.527)\end{array}$ & $\begin{array}{l}0.5311 \\
(-0.7422,1.897)\end{array}$ & $\begin{array}{l}0.4005 \\
(-0.7619,1.667)\end{array}$ & $\begin{array}{l}0.211 \\
(-1.018,1.566)\end{array}$ \\
\hline $\begin{array}{l}-1.045 \\
(-2.358,0.4094)\end{array}$ & $\begin{array}{l}-1.006 \\
(-2.382,0.4138)\end{array}$ & $\begin{array}{l}-1.43 \\
(-3.185,0.3702)\end{array}$ & $\begin{array}{l}-0.6758 \\
(-2.032,0.7361)\end{array}$ & $\begin{array}{l}-0.8102 \\
(-2.062,0.5111)\end{array}$ & $\begin{array}{l}-0.9959 \\
(-2.309,0.4104)\end{array}$ \\
\hline $\begin{array}{l}0.0832 \\
(-0.7052,0.9651)\end{array}$ & $\begin{array}{l}0.1279 \\
(-0.8033,1.022)\end{array}$ & $\begin{array}{l}-0.2993 \\
(-1.721,1.101)\end{array}$ & $\begin{array}{l}0.4611 \\
(-0.3973,1.29)\end{array}$ & $\begin{array}{l}0.3275 \\
(-0.3689,1.012)\end{array}$ & $\begin{array}{l}0.1383 \\
(-0.5344,0.8333)\end{array}$ \\
\hline $\begin{array}{l}0.2829 \\
(-0.4122,1.049)\end{array}$ & $\begin{array}{l}0.3303 \\
(-0.5457,1.13)\end{array}$ & $\begin{array}{l}-0.1026 \\
(-1.49,1.26)\end{array}$ & $\begin{array}{l}0.6588 \\
(-0.1398,1.41)\end{array}$ & $\begin{array}{l}0.5282 \\
(-0.09658, \mathrm{I} . \mathrm{III})\end{array}$ & $\begin{array}{l}0.3383 \\
(-0.2882,0.9599)\end{array}$ \\
\hline $\begin{array}{l}0.001627 \\
(-0.6425,0.7896)\end{array}$ & $\begin{array}{l}0.04756 \\
(-0.5544,0.6626)\end{array}$ & $\begin{array}{l}-0.3816 \\
(-1.713,0.9494)\end{array}$ & $\begin{array}{l}0.3756 \\
(-0.2199,1.007)\end{array}$ & $\begin{array}{l}0.2441 \\
(-0.2438,0.7779)\end{array}$ & $\begin{array}{l}0.05579 \\
(-0.561,0.7437)\end{array}$ \\
\hline
\end{tabular}

Notes: Comparisons should be read from left to right. Log odds ratios $<0$ favor the row-defining treatment. To obtain log odds ratio for comparisons in the opposing direction, negative values should be converted into positive values and vice versa. Significant results are in bold. \begin{tabular}{|l|l|l|l|}
\hline Antipsychotic name & Response rate & All-cause discontinuation \\
\hline
\end{tabular}

Abbreviations: ARI, aripiprazole; BLO, blonanserin; CLO, clozapine; Cloca, clocapramine; HAL, haloperidol; MOS, mosapramine; OLA, olanzapine; PAL, paliperidone; PBO, placebo; PER, perospirone; QUE, quetiapine; RIS, risperidone.

was superior to aripiprazole, blonanserin, clocapramine, haloperidol, mosapramine, and risperidone. Olanzapine was superior to aripiprazole, blonanserin, clocapramine, haloperidol, mosapramine, quetiapine, and risperidone. The use of clozapine was associated with the lowest risk of the outcome, and olanzapine was the second-ranked antipsychotic. No inconsistencies were found (Table 6).

\section{Drowsiness, sedation, and somnolence}

The simple pairwise meta-analysis did not show any significant differences between any of the antipsychotics. In the network meta-analysis, all antipsychotics were inferior to placebo. Aripiprazole, blonanserin, haloperidol, paliperidone, and risperidone were superior to mosapramine. Paliperidone was also superior to clozapine, clocapramine, olanzapine, perospirone, and quetiapine. Blonanserin was superior to perospirone and quetiapine. Risperidone was superior to perospirone. The use of paliperidone was associated with the lowest risk of this outcome, and blonanserin was the second-ranked antipsychotic. No inconsistency was detected (Table 7).

\section{Increased salivation}

Olanzapine was superior to aripiprazole, haloperidol, quetiapine, and risperidone in the pairwise meta-analysis. Quetiapine was superior to mosapramine. In the network meta-analysis, aripiprazole was superior to clozapine and mosapramine. Olanzapine was superior to blonanserin, clozapine, clocapramine, haloperidol, mosapramine, paliperidone, and risperidone. Paliperidone was inferior to placebo. Quetiapine was superior to blonanserin, clocapramine, clozapine, haloperidol, mosapramine, and risperidone. Olanzapine was associated with the lowest risk of this outcome, and quetiapine was the second-ranked antipsychotic. However, there were significant inconsistencies regarding aripiprazole versus olanzapine, and quetiapine versus haloperidol (Table 7).

\section{Extrapyramidal symptoms and the use of anticholinergic drugs/tremor}

Aripiprazole was superior to haloperidol and mosapramine in the pairwise meta-analysis. Blonanserin was superior to haloperidol, and olanzapine was superior to haloperidol 
Table 2 (Continued)

\begin{tabular}{|c|c|c|c|c|c|}
\hline $\begin{array}{l}-1.02 \mid \\
(-1.914,-0.2437)\end{array}$ & $\begin{array}{l}-1.052 \\
(-2.327,0.09627)\end{array}$ & $\begin{array}{l}0.2215 \\
(-1.044,1.364)\end{array}$ & $\begin{array}{l}-0.1648 \\
(-0.9652,0.6276)\end{array}$ & $\begin{array}{l}-0.2858 \\
(-0.9357,0.4231)\end{array}$ & $\begin{array}{l}-0.631 \\
(-1.369,0.04228)\end{array}$ \\
\hline $\begin{array}{l}-0.6514 \\
(-1.541,0.1846)\end{array}$ & $\begin{array}{l}-0.6808 \\
(-1.94,0.5089)\end{array}$ & $\begin{array}{l}0.5946 \\
(-0.6694,1.782)\end{array}$ & $\begin{array}{l}0.2075 \\
(-0.6593,1.117)\end{array}$ & $\begin{array}{l}0.08039 \\
(-0.6679,0.957 I)\end{array}$ & $\begin{array}{l}-0.2584 \\
(-0.866,0.3275)\end{array}$ \\
\hline $\begin{array}{l}-0.676 \\
(-2.376,1.05)\end{array}$ & $\begin{array}{l}-0.7051 \\
(-2.614,1.206)\end{array}$ & $\begin{array}{l}0.5753 \\
(-1.331,2.486)\end{array}$ & $\begin{array}{l}0.1981 \\
(-1.467,1.896)\end{array}$ & $\begin{array}{l}0.07845 \\
(-1.554,1.765)\end{array}$ & $\begin{array}{l}-0.2743 \\
(-1.916,1.401)\end{array}$ \\
\hline $\begin{array}{l}-0.4679 \\
(-1.405,0.4179)\end{array}$ & $\begin{array}{l}-0.4934 \\
(-1.798,0.7247)\end{array}$ & $\begin{array}{l}0.7813 \\
(-0.5198,1.992)\end{array}$ & $\begin{array}{l}0.3961 \\
(-0.4854,1.298)\end{array}$ & $\begin{array}{l}0.2742 \\
(-0.5079, I .127)\end{array}$ & $\begin{array}{l}-0.06984 \\
(-0.7941,0.613)\end{array}$ \\
\hline $\begin{array}{l}-1.009 \\
(-1.733,-0.363)\end{array}$ & $\begin{array}{l}-1.038 \\
(-2.191,0.034)\end{array}$ & $\begin{array}{l}0.2356 \\
(-0.9142,1.305)\end{array}$ & $\begin{array}{l}-0.1486 \\
(-0.8087,0.5274)\end{array}$ & $\begin{array}{l}-0.2727 \\
(-0.8013,0.3524)\end{array}$ & $\begin{array}{l}-0.614 \\
(-1.167,-0.09608)\end{array}$ \\
\hline $\begin{array}{l}-0.9116 \\
(-1.796,-0.1521)\end{array}$ & $\begin{array}{l}-0.9384 \\
(-2.201,0.1936)\end{array}$ & $\begin{array}{l}0.3345 \\
(-0.9236,1.462)\end{array}$ & $\begin{array}{l}-0.05395 \\
(-0.7237,0.599)\end{array}$ & $\begin{array}{l}-0.1766 \\
(-0.7372,0.4416)\end{array}$ & $\begin{array}{l}-0.5205 \\
(-1.224,0.1278)\end{array}$ \\
\hline OLA & $\begin{array}{l}-0.03021 \\
(-0.9131,0.8568)\end{array}$ & $\begin{array}{l}\text { I.246 } \\
(0.3667,2.124)\end{array}$ & $\begin{array}{l}0.8576 \\
(-0.0318,1.852)\end{array}$ & $\begin{array}{l}0.7377 \\
(-0.04115,1.674)\end{array}$ & $\begin{array}{l}0.3928 \\
(-0.3573, I .177)\end{array}$ \\
\hline $\begin{array}{l}-0.01927 \\
(-0.9981,0.9958)\end{array}$ & PAL & $\begin{array}{l}\text { I.275 } \\
(0.5335,2.023)\end{array}$ & $\begin{array}{l}0.889 \\
(-0.3517,2.228)\end{array}$ & $\begin{array}{l}0.7679 \\
(-0.3874,2.08)\end{array}$ & $\begin{array}{l}0.4242 \\
(-0.7241,1.609)\end{array}$ \\
\hline $\begin{array}{l}-1.23 \\
(-2.301,-0.149)\end{array}$ & $\begin{array}{l}-1.205 \\
(-2.157,-0.2846)\end{array}$ & $\mathrm{PBO}$ & $\begin{array}{l}-0.3866 \\
(-1.627,0.947)\end{array}$ & $\begin{array}{l}-0.5037 \\
(-1.644,0.8042)\end{array}$ & $\begin{array}{l}-0.8522 \\
(-1.988,0.3266)\end{array}$ \\
\hline $\begin{array}{l}-0.09214 \\
(-1.104,0.8187)\end{array}$ & $\begin{array}{l}-0.07184 \\
(-1.518,1.262)\end{array}$ & $\begin{array}{l}\text { I.139 } \\
(-0.358,2.538)\end{array}$ & PER & $\begin{array}{l}-0.1239 \\
(-0.9011,0.7267)\end{array}$ & $\begin{array}{l}-0.4668 \\
(-1.328,0.3352)\end{array}$ \\
\hline $\begin{array}{l}0.107 \\
(-0.8154,0.9201)\end{array}$ & $\begin{array}{l}0.1299 \\
(-1.264,1.384)\end{array}$ & $\begin{array}{l}\text { I.335 } \\
(-0.1088,2.678)\end{array}$ & $\begin{array}{l}0.1976 \\
(-0.6648,1.038)\end{array}$ & QUE & $\begin{array}{l}-0.3425 \\
(-1.133,0.3285)\end{array}$ \\
\hline $\begin{array}{l}-0.1758 \\
(-0.9671,0.5755)\end{array}$ & $\begin{array}{l}-0.1525 \\
(-1.451,1.077)\end{array}$ & $\begin{array}{l}\text { I.055 } \\
(-0.2895,2.367)\end{array}$ & $\begin{array}{l}-0.08424 \\
(-0.8914,0.7787)\end{array}$ & $\begin{array}{l}-0.2807 \\
(-0.9795,0.487)\end{array}$ & RIS \\
\hline
\end{tabular}

and risperidone. Quetiapine was superior to haloperidol, mosapramine, and risperidone. In the network meta-analysis, aripiprazole was superior to clocapramine, haloperidol, and mosapramine. Blonanserin was superior to haloperidol. Olanzapine was superior to blonanserin, clocapramine, clozapine, haloperidol, mosapramine, perospirone, and risperidone. In addition, clocapramine, haloperidol, and mosapramine were inferior to placebo. Quetiapine was superior to blonanserin, clocapramine, haloperidol, mosapramine, perospirone, and risperidone. Risperidone was superior to haloperidol and mosapramine. Olanzapine was associated with the lowest risk of this outcome, and quetiapine was the second-ranked antipsychotic. No inconsistency was found between any of the antipsychotics (Table 8).

\section{Akathisia}

Olanzapine was superior to haloperidol and risperidone in the pairwise meta-analysis. Quetiapine was superior to haloperidol. The network meta-analysis showed that aripiprazole was superior to both haloperidol and mosapramine. Clozapine was superior to haloperidol, mosapramine, and perospirone.
Olanzapine was superior to aripiprazole, blonanserin, clocapramine, haloperidol, mosapramine, perospirone, and risperidone. Quetiapine was superior to haloperidol, mosapramine, and perospirone. Risperidone was superior to haloperidol and mosapramine. Olanzapine was associated with the lowest risk of this outcome, and clozapine was the second-ranked antipsychotic. No inconsistency was found (Table 8).

\section{Weight change}

A simple pairwise meta-analysis did not exhibit any significant differences between any of the antipsychotics in terms of this outcome. In the network meta-analysis, blonanserin, haloperidol, placebo, and risperidone were superior to olanzapine. Blonanserin was associated with the lowest risk of the outcome, and aripiprazole was the second-ranked antipsychotic. No inconsistency was detected (Table 9).

\section{Total cholesterol}

The simple pairwise meta-analysis did not show any significant differences between any of the antipsychotics. In the network meta-analysis, aripiprazole was superior 


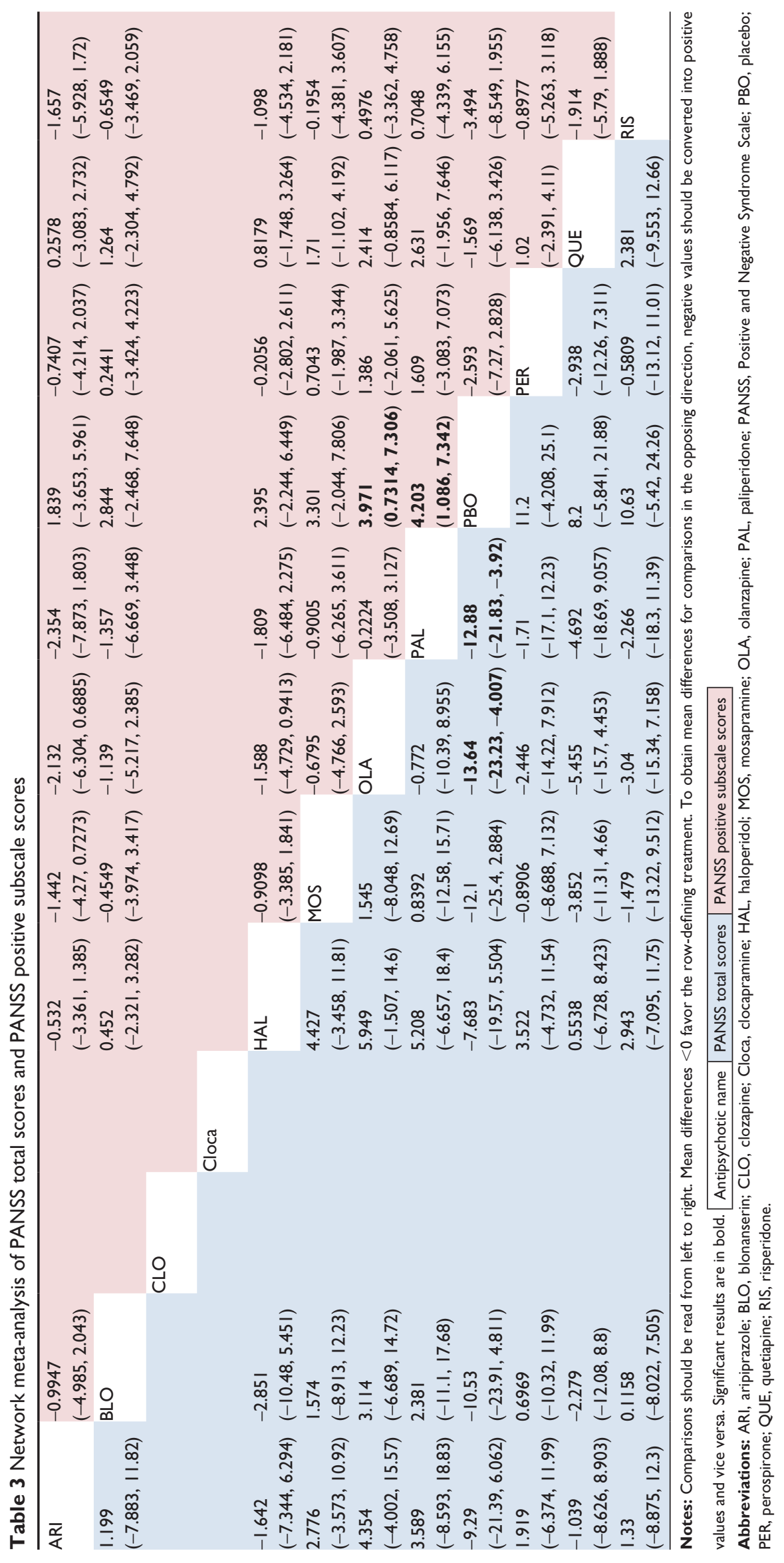




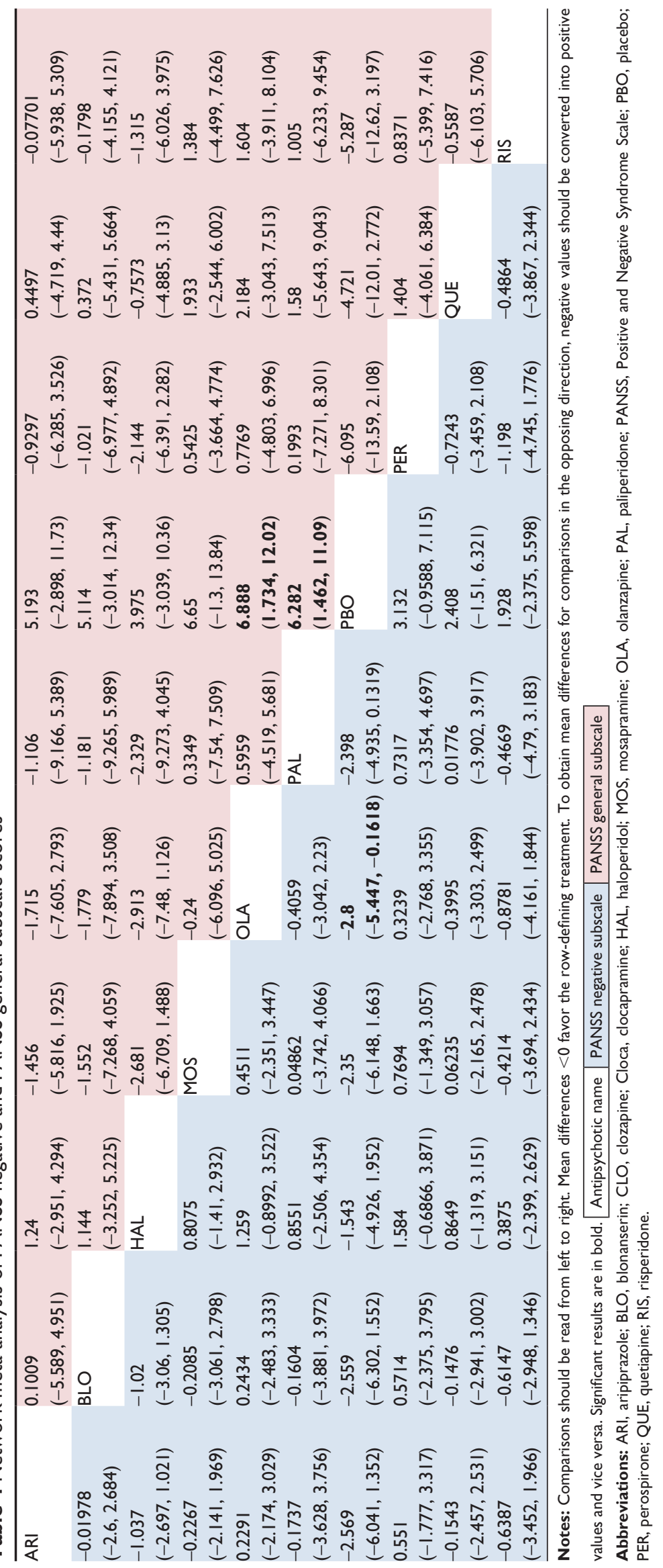


Table 5 Network meta-analysis of discontinuation due to adverse events and at least one adverse event

\begin{tabular}{|c|c|c|c|c|c|}
\hline ARI & $\begin{array}{l}1.207 \\
(-0.5025,3.135)\end{array}$ & & $\begin{array}{l}1.732 \\
(0.3015,3.437)\end{array}$ & $\begin{array}{l}1.53 \\
(0.1822,3.149)\end{array}$ & $\begin{array}{l}I .85 \mathrm{I} \\
(0.5218,3.473)\end{array}$ \\
\hline 0.3651 & BLO & & 0.5249 & 0.3198 & 0.6501 \\
\hline$(-1.021,2.125)$ & & & $(-0.6084,1.736)$ & $(-0.6882, I .4 I)$ & $(-0.4905,1.832)$ \\
\hline-0.5364 & -0.9357 & CLO & & & \\
\hline$(-3.094,1.897)$ & $(-3.60 \mathrm{I}, \mathrm{I} .42)$ & & & & \\
\hline 1.073 & 0.705 & 1.654 & Cloca & -0.2035 & 0.1277 \\
\hline$(-0.402 I, 3.07 I)$ & $(-0.8813,2.484)$ & $(-0.7466,4.444)$ & & $(-0.8267,0.4084)$ & $(-0.5631,0.7513)$ \\
\hline 0.1921 & -0.1696 & 0.7461 & $-0.87 \mid$ & $\mathrm{HAL}$ & 0.3305 \\
\hline$(-0.7213,1.49)$ & $(-1.326,0.989)$ & $(-1.284,3.161)$ & $(-2.343,0.378)$ & & $(-0.25|6,0.857|)$ \\
\hline 0.1105 & -0.2609 & 0.65 & -0.9628 & -0.08889 & MOS \\
\hline$(-0.8998,1.189)$ & $(-1.911,1.098)$ & $(-1.712,3.183)$ & $(-2.833,0.4547)$ & $(-1.228,0.7635)$ & \\
\hline 1.533 & 1.155 & 2.096 & 0.4465 & 1.332 & 1.425 \\
\hline$(0.09354,3.343)$ & $(-0.4025,2.743)$ & $(-0.2767,4.783)$ & $(-1.419,2.139)$ & $(0.1216,2.573)$ & $(0.0096 \mid 5,3.128)$ \\
\hline 1.556 & 1.175 & 2.119 & 0.4536 & 1.355 & $\mathrm{I} .44$ \\
\hline$(-1.082,4.266)$ & $(-1.544,3.725)$ & $(-1.12,5.454)$ & $(-2.431,3.052)$ & $(-1.194,3.693)$ & $(-1.169,4.096)$ \\
\hline 0.5102 & 0.1281 & 1.056 & -0.5845 & 0.3084 & 0.3944 \\
\hline$(-2.041,3.079)$ & $(-2.537,2.539)$ & $(-2.133,4.316)$ & $(-3.422,1.879)$ & $(-2.169,2.494)$ & $(-2.154,2.907)$ \\
\hline 0.7217 & 0.3486 & 1.276 & -0.3501 & 0.5191 & 0.6123 \\
\hline$(-0.6387,2.319)$ & $(-1.364,1.973)$ & $(-1.137,3.93)$ & $(-2.30 \mathrm{I}, \mathrm{I} .297)$ & $(-0.7423,1.702)$ & $(-0.5241,1.923)$ \\
\hline 0.9081 & 0.5469 & 1.467 & -0.1535 & 0.7232 & 0.8037 \\
\hline$(-0.251,2.391)$ & $(-1.046,2.05)$ & $(-0.8523,4.072)$ & $(-1.99,1.37)$ & $(-0.3803,1.718)$ & $(-0.1522,1.99)$ \\
\hline 0.6546 & 0.2898 & 1.214 & -0.4137 & 0.4596 & 0.5509 \\
\hline$(-0.6957,2.291)$ & $(-0.86 / 2,1.392)$ & $(-1.12,3.838)$ & $(-2.079,1.001)$ & $(-0.6572, I .54 I)$ & $(-0.766,2.085)$ \\
\hline
\end{tabular}

Notes: Comparisons should be read from left to right. Log odds ratios $<0$ favor the row-defining treatment. To obtain log odds ratio for comparisons in the opposing direction,

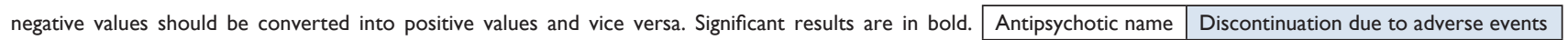
At least one adverse event

Abbreviations: ARI, aripiprazole; BLO, blonanserin; CLO, clozapine; Cloca, clocapramine; HAL, haloperidol; MOS, mosapramine; OLA, olanzapine; PAL, paliperidone; PER, perospirone; PBO, placebo; QUE, quetiapine; RIS, risperidone.

Table 6 Network meta-analysis of agitation/anxiety/use of anxiolytics and insomnia/use of hypnotics

\begin{tabular}{|c|c|c|c|c|c|}
\hline ARI & $\begin{array}{l}0.2366 \\
(-0.4406,0.9037)\end{array}$ & $\begin{array}{l}-1.254 \\
(-2.558,-0.07422)\end{array}$ & $\begin{array}{l}0.1643 \\
(-0.4666,0.8022)\end{array}$ & $\begin{array}{l}0.3403 \\
(-0.1446,0.8133)\end{array}$ & $\begin{array}{l}0.04008 \\
(-0.4627,0.5195)\end{array}$ \\
\hline 0.02746 & BLO & -1.491 & -0.07015 & 0.11 & -0.1942 \\
\hline$(-1.998,2.26)$ & & $(-2.806,-0.3013)$ & $(-0.7229,0.5963)$ & $(-0.402,0.6058)$ & $(-0.8622,0.454 I)$ \\
\hline 2.034 & 2.002 & CLO & 1.421 & 1.597 & 1.292 \\
\hline$(-0.6677,4.998)$ & $(-0.8908,4.96)$ & & $(0.2367,2.738)$ & $(0.5|05,2.8| 2)$ & $(0.1182,2.584)$ \\
\hline 0.751 & 0.7095 & -1.288 & Cloca & 0.1792 & -0.1262 \\
\hline$(-1.059,3.034)$ & $(-1.329,3.057)$ & $(-4.138,1.782)$ & & $(-0.3351,0.668)$ & $(-0.7 \mid 12,0.4387)$ \\
\hline 0.217 & 0.1881 & -1.806 & -0.5223 & HAL & -0.3025 \\
\hline$(-1.155,1.846)$ & $(-1.452,1.864)$ & $(-4.246,0.57 / 9)$ & $(-2.38,1.053)$ & & $(-0.7667,0.1599)$ \\
\hline 0.5329 & 0.5013 & -1.498 & -0.2125 & 0.3129 & MOS \\
\hline$(-0.9177,2.273)$ & $(-1.631,2.717)$ & $(-4.352,1.376)$ & $(-2.177,1.532)$ & $(-1.226,1.894)$ & \\
\hline 0.8269 & 0.7904 & -1.201 & 0.08479 & 0.6043 & 0.2929 \\
\hline$(-0.8 \mid 13,2.884)$ & $(-1.113,2.946)$ & $(-3.962,1.682)$ & $(-1.98 I, 2.06 I)$ & $(-0.7565,2.169)$ & $(-1.545,2.275)$ \\
\hline 0.8315 & 0.8004 & -1.195 & 0.0958 & 0.6132 & 0.3028 \\
\hline$(-1.815,3.927)$ & $(-2.058,3.921)$ & $(-4.668,2.467)$ & $(-2.928,3.027)$ & $(-1.926,3.357)$ & $(-2.529,3.302)$ \\
\hline 0.4778 & 0.449 & -1.547 & -0.2544 & 0.2623 & -0.04831 \\
\hline$(-2.163,3.578)$ & $(-2.421,3.56)$ & $(-5.021,2.129)$ & $(-3.289,2.68)$ & $(-2.272,3.007)$ & $(-2.884,2.937)$ \\
\hline 0.6266 & 0.5944 & -1.406 & -0.1165 & 0.406 & 0.09424 \\
\hline$(-1.36,2.874)$ & $(-1.803,3.063)$ & $(-4.432,1.629)$ & $(-2.54,2.06 \mathrm{I})$ & $(-1.433,2.27)$ & $(-1.701,1.864)$ \\
\hline 0.9923 & 0.9475 & -1.037 & 0.2452 & 0.7675 & 0.4503 \\
\hline$(-0.4666,3.024)$ & $(-0.9511,3.269)$ & $(-3.731,1.921)$ & $(-1.718,2.269)$ & $(-0.5429,2.42 \mathrm{I})$ & $(-0.9938,2.207)$ \\
\hline 0.2535 & 0.2251 & -1.774 & -0.4923 & 0.03448 & -0.2762 \\
\hline$(-1.396,2.081)$ & $(-1.456,1.879)$ & $(-4.559,0.9095)$ & $(-2.356,1.062)$ & $(-1.28,1.295)$ & $(-2.1,1.449)$ \\
\hline
\end{tabular}

Notes: Comparisons should be read from left to right. Log odds ratios $<0$ favor the row-defining treatment. To obtain log odds ratio for comparisons in the opposing

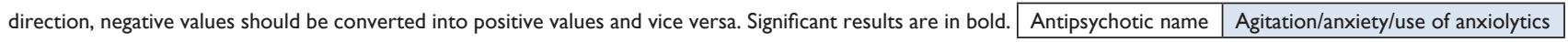
Insomnia/use of hypnotics

Abbreviations: ARI, aripiprazole; BLO, blonanserin; CLO, clozapine; Cloca, clocapramine; HAL, haloperidol; MOS, mosapramine; OLA, olanzapine; PAL, paliperidone; PBO, placebo; PER, perospirone; QUE, quetiapine; RIS, risperidone. 
Table 5 (Continued)

\begin{tabular}{|c|c|c|c|c|c|}
\hline 1.255 & 0.9555 & 0.8506 & 1.399 & 0.7549 & 1.125 \\
\hline$(-0.3619,3.143)$ & $(-1.024,3.156)$ & $(-1.143,3.047)$ & $(-0.08294,3.137)$ & $(-0.7186,2.482)$ & $(-0.360 I, 2.869)$ \\
\hline 0.04347 & -0.2568 & -0.3612 & 0.1907 & -0.4548 & -0.09088 \\
\hline$(-1.3 \mid 1,1.472)$ & $(-2.036,1.57)$ & $(-2.156,1.448)$ & $(-1.05,1.482)$ & $(-1.698,0.848)$ & $(-1.18,1.09)$ \\
\hline-0.4764 & -0.7877 & -0.889 & -0.3358 & -0.9825 & -0.6185 \\
\hline$(-1.599,0.6216)$ & $(-2.38 \mathrm{I}, 0.793)$ & $(-2.48,0.6792)$ & $(-1.242,0.5339)$ & $(-1.88,-0.1021)$ & $(-1.308,0.09244)$ \\
\hline-0.2776 & -0.5842 & -0.6838 & -0.1353 & -0.7763 & -0.4187 \\
\hline$(-1.206,0.6459)$ & $(-2.06 \mathrm{I}, 0.8745)$ & $(-2.164,0.7625)$ & $(-0.8779,0.59 \mid 4)$ & $(-1.52,-0.05692)$ & $(-1.095,0.2883)$ \\
\hline-0.6086 & -0.9169 & -1.013 & -0.4614 & -1.107 & -0.7466 \\
\hline$(-1.662,0.4926)$ & $(-2.463,0.6784)$ & $(-2.565,0.5602)$ & $(-1.189,0.2863)$ & $(-1.827,-0.3555)$ & $(-1.518,0.109)$ \\
\hline \multirow[t]{2}{*}{ OLA } & -0.3023 & -0.4043 & 0.1435 & -0.5012 & -0.1421 \\
\hline & $(-1.47,0.8099)$ & $(-1.574,0.6966)$ & $(-1.052,1.316)$ & $(-1.685,0.6742)$ & $(-1.273,1.036)$ \\
\hline 0.03276 & PAL & -0.1033 & $0.447 \mid$ & -0.1952 & 0.1667 \\
\hline$(-2.23,2.013)$ & & $(-1.021,0.8077)$ & $(-1.2,2.085)$ & $(-1.832,1.445)$ & $(-1.427,1.8)$ \\
\hline-1.013 & -1.041 & PBO & 0.5505 & -0.09577 & 0.269 \\
\hline$(-3.204,0.7688)$ & $(-2.67,0.456)$ & & $(-1.083,2.185)$ & $(-1.724,1.547)$ & $(-1.317,1.904)$ \\
\hline-0.81 & -0.82 & 0.2256 & PER & -0.6445 & -0.2818 \\
\hline$(-2.58 \mathrm{I}, 0.8572)$ & $(-3.511,1.929)$ & $(-2.328,2.907)$ & & $(-1.601,0.3261)$ & $(-1.235,0.7192)$ \\
\hline-0.6091 & -0.6325 & 0.4149 & 0.1965 & QUE & 0.3597 \\
\hline$(-2.25 \mathrm{I}, 0.9353)$ & $(-3.233,2.077)$ & $(-2.04,3.067)$ & $(-1.243,1.669)$ & & $(-0.592,1.365)$ \\
\hline-0.8687 & -0.8924 & 0.1537 & -0.0689 & -0.2561 & RIS \\
\hline$(-2.33,0.5105)$ & $(-3.368,1.727)$ & $(-2.173,2.737)$ & $(-1.652,1.576)$ & $(-1.724,1.233)$ & \\
\hline
\end{tabular}

Table 6 (Continued)

\begin{tabular}{|c|c|c|c|c|c|}
\hline-0.93 & -0.6482 & -0.8565 & -0.1123 & -0.06873 & 0.07909 \\
\hline$(-1.744,-0.163 I)$ & $(-2.075,0.9095)$ & $(-2.325,0.7172)$ & $(-0.8592,0.6095)$ & $(-0.7297,0.5379)$ & $(-0.53|6,0.7| 4)$ \\
\hline-1.165 & -0.8766 & -1.082 & -0.3399 & -0.3048 & -0.1539 \\
\hline$(-2.01,-0.3486)$ & $(-2.347,0.6808)$ & $(-2.589,0.5 \mid 34)$ & $(-1.172,0.4436)$ & $(-1.04,0.4049)$ & $(-0.6391,0.3555)$ \\
\hline 0.3258 & 0.6197 & $0.4 I 28$ & I.146 & 1.19 & 1.34 \\
\hline$(-0.9473,1.691)$ & $(-1.15|, 2.5| 6)$ & $(-1.37,2.333)$ & $(-0.1292,2.494)$ & $(-0.0507,2.522)$ & $(0.1624,2.647)$ \\
\hline-1.099 & -0.8153 & -1.022 & -0.274 & -0.2355 & -0.08557 \\
\hline$(-1.941,-0.3003)$ & $(-2.252,0.7605)$ & $(-2.506,0.574 I)$ & $(-1.059,0.4875)$ & $(-0.9506,0.434)$ & $(-0.664 I, 0.5068)$ \\
\hline$-|.27|$ & -0.9894 & -1.197 & -0.4517 & -0.4132 & -0.2615 \\
\hline$(-1.963,-0.627 I)$ & $(-2.356,0.5219)$ & $(-2.597,0.3063)$ & $(-1.109,0.1851)$ & $(-0.9681,0.1203)$ & $(-0.7286,0.2328)$ \\
\hline$-0.97 I I$ & -0.6878 & -0.9001 & -0.1501 & -0.1089 & 0.04117 \\
\hline$(-1.77 \mid,-0.2002)$ & $(-2.111,0.8699)$ & $(-2.34 I, 0.679 I)$ & $(-0.7901,0.4817)$ & $(-0.6954,0.4524)$ & $(-0.552 I, 0.6688)$ \\
\hline \multirow[t]{2}{*}{ OLA } & 0.2804 & 0.0773 & 0.8212 & 0.8612 & 1.01 \\
\hline & $(-0.9069,1.646)$ & $(-1.135,1.428)$ & $(-0.09131,1.739)$ & $(0.03656,1.689)$ & $(0.2543, I .838)$ \\
\hline 0.01236 & PAL & -0.2072 & 0.5332 & 0.5746 & 0.7237 \\
\hline$(-2.18,2.21 \mathrm{I})$ & & $(-1.131,0.7014)$ & $(-1.086,2.035)$ & $(-1.009,2.021)$ & $(-0.8208,2.177)$ \\
\hline-0.3407 & -0.3518 & PBO & 0.7475 & 0.7776 & 0.929 \\
\hline$(-2.532,1.856)$ & $(-2.493,1.793)$ & & $(-0.9019,2.264)$ & $(-0.7983,2.265)$ & $(-0.634 \mathrm{I}, 2.409)$ \\
\hline-0.2012 & -0.2106 & 0.1396 & PER & 0.04342 & 0.1928 \\
\hline$(-2.521,1.964)$ & $(-3.45 I, 2.834)$ & $(-3.086,3.188)$ & & $(-0.7497,0.824 I)$ & $(-0.569,0.9953)$ \\
\hline 0.1591 & 0.1436 & 0.4936 & 0.3607 & QUE & 0.1517 \\
\hline$(-1.51,1.993)$ & $(-2.58,3.034)$ & $(-2.22,3.388)$ & $(-1.597,2.647)$ & & $(-0.5139,0.8521)$ \\
\hline-0.5731 & -0.5781 & -0.2253 & -0.3733 & -0.7346 & RIS \\
\hline$(-2.192,0.8174)$ & $(-3.366,1.95)$ & $(-3.021,2.302)$ & $(-2.544,1.725)$ & $(-2.615,0.762)$ & \\
\hline
\end{tabular}


Table 7 Network meta-analysis of drowsiness/sedation/somnolence and increased salivation

\begin{tabular}{|c|c|c|c|c|c|}
\hline ARI & $\begin{array}{l}0.606 \mathrm{I} \\
(-0.6003,1.75)\end{array}$ & $\begin{array}{l}2.025 \\
(0.2981,3.693)\end{array}$ & $\begin{array}{l}0.8816 \\
(-0.3772,2.127)\end{array}$ & $\begin{array}{l}0.8949 \\
(-0.03768,1.719)\end{array}$ & $\begin{array}{l}1.284 \\
(0.393,2.166)\end{array}$ \\
\hline 0.2048 & BLO & 1.418 & 0.2709 & 0.2932 & 0.6743 \\
\hline$(-0.955 \mathrm{I}, \mathrm{I} .4 \mathrm{I} 8)$ & & $(-0.2763,3.101)$ & $(-0.9826,1.587)$ & $(-0.6358, I . \mid 4 I)$ & $(-0.4175,1.831)$ \\
\hline-0.9653 & -1.173 & CLO & -1.149 & -1.13 & -0.7395 \\
\hline$(-2.45,0.4876)$ & $(-2.502,0.1338)$ & & $(-2.943,0.7163)$ & $(-2.602,0.295 \mathrm{I})$ & $(-2.373,0.9443)$ \\
\hline-0.7438 & -0.9473 & 0.219 & Cloca & 0.01736 & 0.3982 \\
\hline$(-1.95 I, 0.45 \mid 4)$ & $(-2.021,0.03385)$ & $(-1.17,1.599)$ & & $(-1.168,1.089)$ & $(-0.6654,1.479)$ \\
\hline-0.3594 & -0.5689 & 0.6025 & 0.382 & $\mathrm{HAL}$ & 0.3855 \\
\hline$(-1.296,0.5911)$ & $(-1.302,0.1555)$ & $(-0.475, I .7 I I)$ & $(-0.4341,1.257)$ & & $(-0.3629,1.248)$ \\
\hline-1.082 & -1.29 & -0.1215 & -0.3348 & -0.7264 & MOS \\
\hline$(-2.059,-0.13)$ & $(-2.298,-0.2943)$ & $(-1.431,1.198)$ & $(-1.298,0.6366)$ & $(-1.444,-0.01546)$ & \\
\hline-0.7512 & -0.9566 & 0.2118 & -0.009993 & -0.3878 & 0.3323 \\
\hline$(-2.386,0.7863)$ & $(-2.495,0.4513)$ & $(-1.525,1.865)$ & $(-1.56 I, 1.5)$ & $(-1.733,0.8304)$ & $(-1.195,1.752)$ \\
\hline 1.508 & 1.301 & 2.474 & 2.256 & 1.865 & 2.591 \\
\hline$(-0.7104,3.845)$ & $(-0.813,3.559)$ & $(0.2147,4.884)$ & $(0.1182,4.559)$ & $(-0.1412,4.029)$ & $(0.465,4.877)$ \\
\hline 44.67 & 44.5 & 45.74 & 45.44 & 45.06 & 45.82 \\
\hline $\begin{array}{l}(6.078,180) \\
-|.15|\end{array}$ & $\begin{array}{l}(5.824,179.6) \\
-1.359\end{array}$ & $\begin{array}{l}(6.992,180.8) \\
-0.1876\end{array}$ & $\begin{array}{l}(\mathbf{6 . 8 1} \mathbf{I}, \mathbf{1 8 0 . 6}) \\
-0.4065\end{array}$ & $\begin{array}{l}(6.379, \mathbf{1 8 0 . 2}) \\
-0.7932\end{array}$ & $\begin{array}{l}(\mathbf{7 . 0 9 3}, \mathbf{1 8 0 . 9 )} \\
-0.05985\end{array}$ \\
\hline$(-2.42,0.09567)$ & $(-2.616,-0.1507)$ & $(-1.694,1.292)$ & $(-1.642,0.8308)$ & $(-1.817,0.1959)$ & $(-1.048,0.8614)$ \\
\hline-0.8991 & $-I . I I$ & 0.06537 & -0.1564 & -0.5389 & 0.1825 \\
\hline$(-2.08,0.2733)$ & $(-2.218,-0.01571)$ & $(-1.311,1.438)$ & $(-1.267,0.9968)$ & $(-1.381,0.2774)$ & $(-0.7054,1.079)$ \\
\hline 0.1775 & -0.02981 & 1.141 & 0.9201 & 0.5378 & 1.266 \\
\hline$(-1.006,1.389)$ & $(-0.7439,0.6799)$ & $(-0.1756,2.483)$ & $(-0.01637,1.938)$ & $(-0.2216,1.295)$ & $(0.2693,2.272)$ \\
\hline
\end{tabular}

Notes: Comparisons should be read from left to right. Log odds ratios $<0$ favor the row-defining treatment. To obtain log odds ratio for comparisons in the opposing direction, negative \begin{tabular}{|l|l|l|l|} 
values should be converted into positive values and vice versa. Significant results are in bold. Antipsychotic name & Drowsiness/sedation/somnolence & Increased salivation \\
\hline
\end{tabular} Abbreviations: ARI, aripiprazole; BLO, blonanserin; CLO, clozapine; Cloca, clocapramine; HAL, haloperidol; MOS, mosapramine; OLA, olanzapine; PAL, paliperidone; PER, perospirone; PBO, placebo; QUE, quetiapine; RIS, risperidone.

Table 8 Network meta-analysis of extrapyramidal symptoms/use of anticholinergic drugs/tremor and akathisia

\begin{tabular}{|c|c|c|c|c|c|}
\hline ARI & $\begin{array}{l}0.2408 \\
(-0.54 \mid 4,1.105)\end{array}$ & $\begin{array}{l}-0.8997 \\
(-2.269,0.3868)\end{array}$ & $\begin{array}{l}0.1721 \\
(-0.5945,0.9705)\end{array}$ & $\begin{array}{l}0.6042 \\
(0.02994,1.209)\end{array}$ & $\begin{array}{l}0.7682 \\
(0.153,1.402)\end{array}$ \\
\hline-0.4683 & BLO & -1.156 & -0.07104 & 0.3631 & 0.5273 \\
\hline$(-1.259,0.2373)$ & & $(-2.534,0.1166)$ & $(-0.8832,0.7058)$ & $(-0.2795,0.946)$ & $(-0.297,1.287)$ \\
\hline-0.7214 & -0.2499 & CLO & 1.081 & 1.509 & 1.674 \\
\hline$(-1.872,0.4 \mid 44)$ & $(-1.405,0.9509)$ & & $(-0.2017,2.453)$ & $(0.3777,2.75)$ & $(0.4221,3.03)$ \\
\hline-0.858 & -0.386 & -0.1365 & Cloca & 0.4331 & 0.5959 \\
\hline$(-1.559,-0.2091)$ & $(-1.08 I, 0.324)$ & $(-1.299,0.9979)$ & & $(-0.1706,1.031)$ & $(-0.0966,1.282)$ \\
\hline-1.107 & -0.6395 & -0.3887 & -0.2547 & HAL & 0.1639 \\
\hline$(-1.65,-0.6179)$ & $(-1.188,-0.06049)$ & $(-1.43,0.626 I)$ & $(-0.7616,0.2702)$ & & $(-0.3778,0.7015)$ \\
\hline-1.164 & -0.6963 & -0.439 & -0.3069 & -0.05563 & MOS \\
\hline$(-1.7 I I,-0.6402)$ & $(-1.361,0.02994)$ & $(-1.564,0.6721)$ & $(-0.8564,0.268 I)$ & $(-0.4835,0.4043)$ & \\
\hline 0.5232 & 0.9951 & 1.243 & 1.379 & 1.632 & 1.688 \\
\hline $\begin{array}{l}(-0.274,1.265) \\
-0.1609\end{array}$ & $\begin{array}{l}(0.1826,1.807) \\
0.3148\end{array}$ & $\begin{array}{l}(0.02601,2.437) \\
0.5532\end{array}$ & $\begin{array}{l}(0.6054,2.154) \\
0.6968\end{array}$ & $\begin{array}{l}(0.9908,2.265) \\
0.9514\end{array}$ & $\begin{array}{l}(\mathbf{0 . 9 3 2 6}, \mathbf{2 . 4 2}) \\
1.005\end{array}$ \\
\hline$(-1.633,1.167)$ & $(-1.172,1.703)$ & $(-1.153,2.197)$ & $(-0.7591,2.059)$ & $(-0.4424,2.23)$ & $(-0.447 \mid, 2.33 I)$ \\
\hline 0.6894 & 1.166 & 1.408 & 1.55 & 1.804 & 1.858 \\
\hline$(-0.8322,2.095)$ & $(-0.3713,2.62)$ & $(-0.3474,3.104)$ & $(0.03722,2.986)$ & $(0.3486,3.155)$ & $(0.3534,3.258)$ \\
\hline-0.6801 & -0.2105 & 0.04402 & 0.1766 & 0.4303 & 0.4842 \\
\hline$(-1.448,0.05674)$ & $(-1.014,0.6503)$ & $(-1.167,1.24 I)$ & $(-0.5706,0.9567)$ & $(-0.1855,1.065)$ & $(-0.1405,1.104)$ \\
\hline 0.4007 & 0.87 I & 1.121 & 1.256 & 1.509 & 1.563 \\
\hline$(-0.2625,1.079)$ & $(0.1437,1.693)$ & $(-0.02032,2.308)$ & $(0.594,1.997)$ & $(0.983,2.096)$ & $(1.017,2.158)$ \\
\hline-0.5164 & -0.04225 & 0.2005 & 0.3425 & 0.5928 & 0.6478 \\
\hline$(-1.231,0.08419)$ & $(-0.635,0.482)$ & $(-0.9691,1.299)$ & $(-0.2518,0.8668)$ & $(0.07963,1.033)$ & $(-3.823 e-05,-1.202)$ \\
\hline
\end{tabular}

Notes: Comparisons should be read from left to right. Log odds ratios $<0$ favors the row-defining treatment. To obtain log odds ratio for comparisons in the opposing direction, negative values should be converted into positive values and vice versa. Significant results are in bold. Antipsychotic name Extrapyramidal symptoms/use of anticholinergic drugs/tremor Akathisia

Abbreviations: ARI, aripiprazole; BLO, blonanserin; CLO, clozapine; Cloca, clocapramine; HAL, haloperidol; MOS, mosapramine; OLA, olanzapine; PAL, paliperidone; PBO, placebo; PER, perospirone; QUE, quetiapine; RIS, risperidone. 
Table 7 (Continued)

\begin{tabular}{|c|c|c|c|c|c|}
\hline-1.183 & 23.91 & -23.33 & 0.2845 & -0.8366 & 0.5972 \\
\hline$(-2.812,0.1217)$ & $(-1.126,67.19)$ & $(-108,34.38)$ & $(-1.099,1.565)$ & $(-2.178,0.2133)$ & $(-0.4044,1.617)$ \\
\hline-1.78 & 23.34 & -23.92 & -0.3232 & -1.439 & -0.01484 \\
\hline$(-3.4 I I,-0.4936)$ & $(-1.709,66.5 I)$ & $(-108.7,33.82)$ & $(-1.762,1.077)$ & $(-2.859,-0.2946)$ & $(-0.8584,0.9143)$ \\
\hline-3.214 & 21.89 & -25.33 & -1.749 & -2.865 & -1.429 \\
\hline$(-5.242,-1.426)$ & $(-3.066,65.22)$ & $(-109.9,32.37)$ & $(-3.612,0.1021)$ & $(-4.735,-1.196)$ & $(-3.04,0.2412)$ \\
\hline-2.059 & 23.07 & -24.18 & -0.5992 & -1.72 & -0.2833 \\
\hline$(-3.83,-0.6426)$ & $(-2.036,66.29)$ & $(-108.9,33.55)$ & $(-2.123,0.8576)$ & $(-3.242,-0.4825)$ & $(-1.321,0.7568)$ \\
\hline-2.07 & 23.05 & -24.2 & -0.6144 & -1.728 & -0.3024 \\
\hline$(-3.461,-0.9683)$ & $(-2.005,66.27)$ & $(-108.9,33.55)$ & $(-1.764,0.5487)$ & $(-2.827,-0.837 I)$ & $(-1.015,0.5382)$ \\
\hline-2.462 & 22.62 & -24.6 & -0.9973 & -2.118 & -0.6853 \\
\hline$(-4.073,-1.199)$ & $(-2.449,65.88)$ & $(-109.3,33.17)$ & $(-2.212,0.1168)$ & $(-3.354,-1.175)$ & $(-1.638,0.2733)$ \\
\hline \multirow[t]{2}{*}{ OLA } & 25.19 & -22.13 & 1.47 & 0.3367 & I.774 \\
\hline & $(0.2008,68.46)$ & $(-106.7,35.64)$ & $(-0.115,3.26)$ & $(-1.109,1.903)$ & $(0.6266,3.288)$ \\
\hline 2.25 & PAL & -43.64 & -23.61 & -24.7 & -23.36 \\
\hline$(0.7916,4.057)$ & & $(-150.5,-2.2)$ & $(-66.9,1.423)$ & $(-67.98,0.2306)$ & $(-66.57,1.685)$ \\
\hline 45.4 & 43.11 & PBO & 23.61 & 22.46 & 23.9 \\
\hline$(6.74,180.7)$ & $(4.567,178.3)$ & & $(-34.17,108.2)$ & $(-35.36,107.1)$ & $(-33.8,108.6)$ \\
\hline-0.3975 & -2.657 & -45.87 & PER & -1.117 & 0.3134 \\
\hline$(-2.005,1.301)$ & $(-5.036,-0.4268)$ & $(-180.9,-7.167)$ & & $(-2.656,0.2343)$ & $(-0.9663,1.692)$ \\
\hline-0.1514 & -2.412 & -45.59 & 0.2491 & QUE & $\mid .43$ I \\
\hline$(-1.635,1.447)$ & $(-4.738,-0.2368)$ & $(-180.6,-6.911)$ & $(-0.9467,1.484)$ & & $(0.4243,2.747)$ \\
\hline 0.9232 & -1.328 & -44.54 & 1.326 & 1.078 & RIS \\
\hline$(-0.4985,2.458)$ & $(-3.593,0.7999)$ & $(-179.6,-5.749)$ & $(0.1084,2.601)$ & $(-0.02801,2.191)$ & \\
\hline
\end{tabular}

Table 8 (Continued)

\begin{tabular}{|c|c|c|c|c|c|}
\hline-1.017 & 6.518 & 5.584 & 0.4952 & -0.5174 & -0.1042 \\
\hline$(-1.97,-0.08602)$ & $(-1.882,29.6 I)$ & $(-3.401,29.1)$ & $(-0.3378, I .35 \mathrm{I})$ & $(-I .357,0.288 I)$ & $(-0.8096,0.6807)$ \\
\hline$-1.26 \mid$ & 6.248 & 5.325 & 0.2522 & -0.7595 & -0.3487 \\
\hline$(-2.262,-0.3537)$ & $(-2.126,29.31)$ & $(-3.643,28.8)$ & $(-0.6981,1.134)$ & $(-1.726,0.09475)$ & $(-0.9527,0.2825)$ \\
\hline-0.1098 & 7.391 & 6.507 & 1.402 & 0.3878 & 0.8025 \\
\hline$(-1.504,1.34)$ & $(-1.132,30.48)$ & $(-2.648,29.94)$ & $(0.05793,2.825)$ & $(-0.9815,1.791)$ & $(-0.4196,2.159)$ \\
\hline-1.19 & 6.331 & 5.43 & 0.3222 & -0.6935 & -0.2749 \\
\hline$(-2.172,-0.2635)$ & $(-2.12,29.39)$ & $(-3.594,28.9)$ & $(-0.5526, \mathrm{I} .183)$ & $(-1.594,0.1538)$ & $(-0.9222,0.418)$ \\
\hline-1.62 & 5.895 & 4.982 & -0.113 & $-I .122$ & -0.7074 \\
\hline$(-2.436,-0.8637)$ & $(-2.455,28.95)$ & $(-3.99,28.45)$ & $(-0.8155,0.5886)$ & $(-1.856,-0.448)$ & $(-1.217,-0.1396)$ \\
\hline-1.783 & 5.739 & 4.819 & -0.2752 & -1.287 & -0.8703 \\
\hline$(-2.734,-0.8784)$ & $(-2.658,28.82)$ & $(-4.162,28.31)$ & $(-0.9771,0.4219)$ & $(-2.04,-0.582 I)$ & $(-1.56,-0.1284)$ \\
\hline \multirow[t]{2}{*}{ OLA } & 7.514 & 6.613 & 1.506 & 0.4966 & 0.9161 \\
\hline & $(-0.7899,30.58)$ & $(-2.314,30.14)$ & $(0.4879,2.566)$ & $(-0.5255,1.526)$ & $(0.0908 \mathrm{I}, \mathrm{I} .827)$ \\
\hline-0.6789 & $\mathrm{PAL}$ & -0.8985 & -5.999 & -7.019 & -6.605 \\
\hline$(-1.914,0.4359)$ & & $(-4.743,1.756)$ & $(-29.08,2.414)$ & $(-30.12,1.415)$ & $(-29.65,1.782)$ \\
\hline 0.1715 & 0.847 & PBO & -5.102 & -6.108 & -5.689 \\
\hline$(-I . I I 5, I .374)$ & $(-0.0404, I .781)$ & & $(-28.61,3.894)$ & $(-29.6,2.894)$ & $(-29.13,3.267)$ \\
\hline-1.205 & -0.5215 & $-1.37 \mid$ & PER & $-1.01 \mathrm{I}$ & -0.5978 \\
\hline$(-2.063,-0.3046)$ & $(-1.922,1)$ & $(-2.849,0.1994)$ & & $(-1.957,-0.104)$ & $(-1.426,0.2999)$ \\
\hline-0.1194 & 0.5599 & -0.2875 & $\mathrm{I} .079$ & QUE & 0.4165 \\
\hline$(-0.9014,0.71)$ & $(-0.7845,2.064)$ & $(-1.717,1.259)$ & $(0.3237,1.883)$ & & $(-0.3729,1.315)$ \\
\hline-1.039 & -0.3621 & -1.217 & 0.1628 & -0.9155 & RIS \\
\hline$(-1.77,-0.36)$ & $(-1.706,1.048)$ & $(-2.626,0.2533)$ & $(-0.6548,0.890 I)$ & $(-1.674,-0.2836)$ & \\
\hline
\end{tabular}




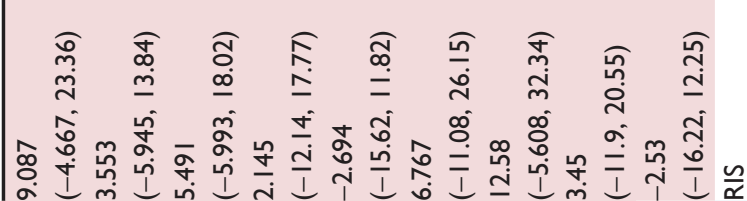

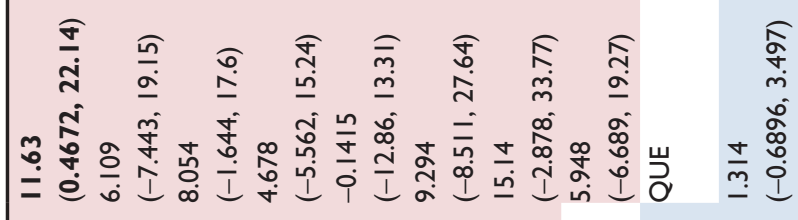

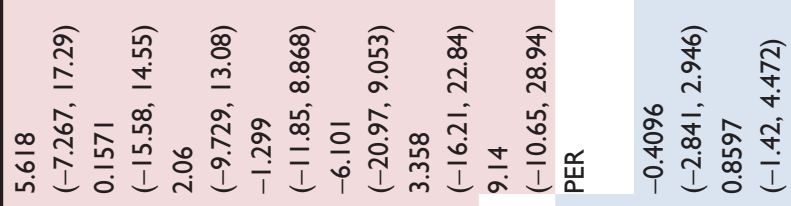

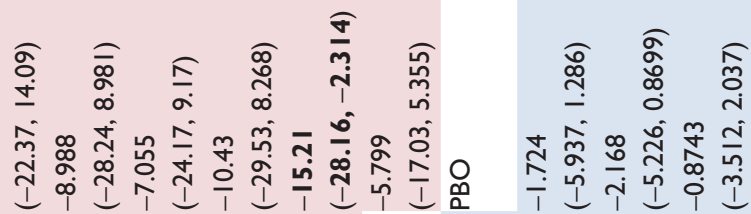

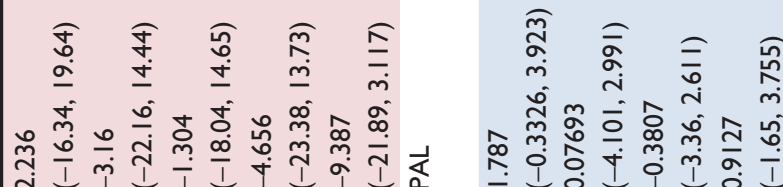

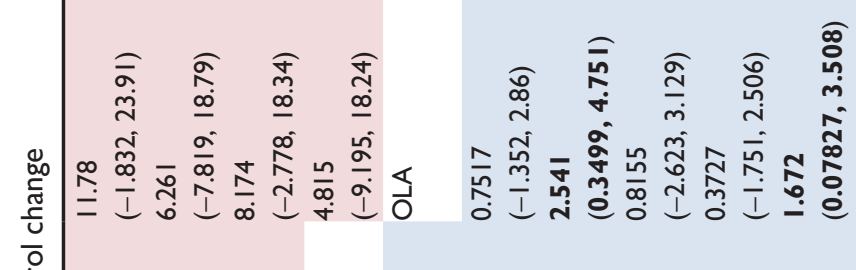

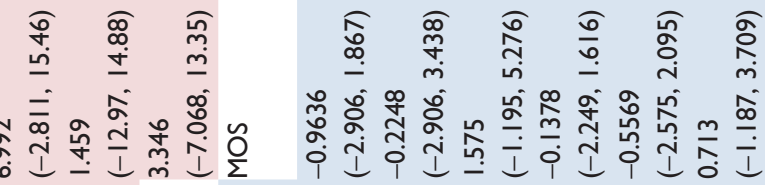

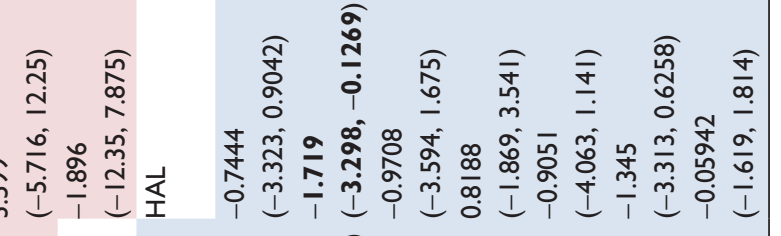

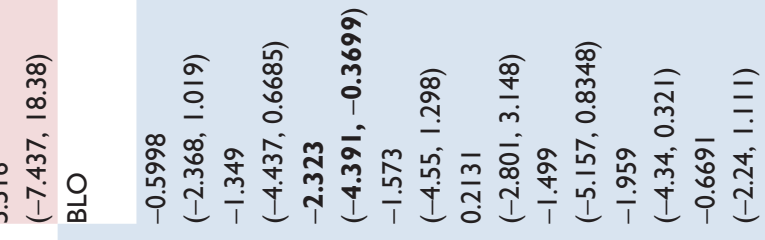

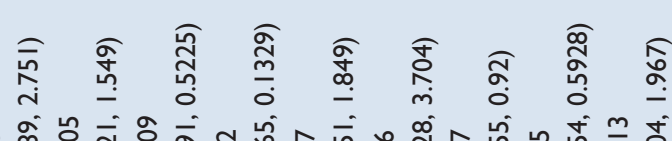

œ

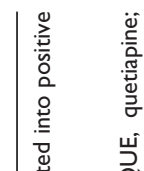

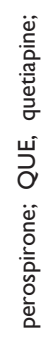

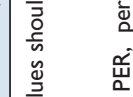

$\ddot{2}$
$\ddot{0}$
$\stackrel{0}{0}$

i

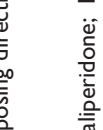

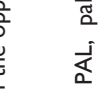

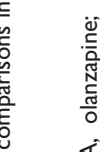

ᄒ 
to quetiapine, and olanzapine was inferior to placebo. Paliperidone was associated with the lowest risk of this outcome, and aripiprazole was the second-ranked antipsychotic. No inconsistency was detected (Table 9).

\section{Triglyceride}

Neither the simple pairwise meta-analysis nor the network meta-analysis showed any significant differences between any of the antipsychotics. Mosapramine was associated with the lowest risk of this outcome, and aripiprazole was the second-ranked antipsychotic. No inconsistency was detected (Table 10).

\section{Potentially prolactin-related adverse events}

Aripiprazole was superior to haloperidol and mosapramine in the pairwise meta-analysis. Clocapramine was superior to mosapramine, and quetiapine was superior to haloperidol. Haloperidol was superior to risperidone. The network metaanalysis showed that aripiprazole was superior to blonanserin, clocapramine, clozapine, haloperidol, mosapramine, perospirone, and risperidone. Quetiapine was superior to blonanserin, clozapine, clocapramine, haloperidol, mosapramine, perospirone, and risperidone. Quetiapine was associated with the lowest risk of the outcome, followed by aripiprazole. Significant inconsistencies were detected between clocapramine and mosapramine, as well as between risperidone and haloperidol (Table 10).

\section{Discussion}

This is the first network meta-analysis of antipsychotics regarding the efficacy and acceptability/safety of SGAs, including aripiprazole, blonanserin, clozapine, clocapramine, mosapramine, olanzapine, paliperidone, perospirone, quetiapine, and risperidone. To maximize sample homogeneity, Japanese samples were exclusively included. The results are in agreement with those of a previous study. ${ }^{1}$ For the primary outcomes of response rate and all-cause discontinuation, paliperidone and olanzapine showed significant superiority over placebo, whereas placebo was ranked the lowest. Although the efficacy subscales were consistent with the primary outcome and with those in the previous study, the safety subscales demonstrated diverse results.

The current network meta-analysis demonstrated that olanzapine and paliperidone were significantly superior to placebo in the outcome of all-cause discontinuation. Olanzapine was also superior to aripiprazole, haloperidol, and mosapramine in both the all-cause discontinuation and discontinuation due to adverse events outcomes. This is consistent with a previous meta-analysis. ${ }^{1}$ Clinically important differences for short-term treatment seem to exist between commonly prescribed antipsychotics in both the efficacy and acceptability of olanzapine and paliperidone.

Although these results were not contradictory to those of the previous study, there were several inconsistencies. The previous meta-analysis found that all antipsychotics were superior to placebo for the overall change in symptoms. Moreover, clozapine had the largest effect size relative to that of placebo, and olanzapine (third-ranked), as well as paliperidone (fifth-ranked), had a moderate effect size compared with that of placebo. ${ }^{1}$ In the current meta-analysis, only olanzapine and paliperidone were significantly more effective than placebo for the response rate, as well as in the improvement of the PANSS total and PANSS positive subscale scores. Placebo was ranked the lowest in these efficacy outcomes with large probabilities $(\sim \geq 70 \%)$. Although all drugs exhibited a significant superiority over placebo in the previous study, only olanzapine and paliperidone showed significant superiorities in the current study. The following reasons may account for this inconsistency. Firstly, the number of studies included in the meta-analysis was small; therefore, the current analysis had insufficient statistical power in addition to the fact that most studies included in this meta-analysis were non-inferiority trials. Secondly, the placebo-controlled study in the current analysis had a short period of 6 weeks; thus, the placebo effect may have been large enough to influence the effect size. Thirdly, only one placebo study was included in the current analysis, although Bayesian inference may compensate for the weaker comparison to a certain extent. And fourthly, because the patients in the placebo arm used antipsychotics, benzodiazepines, or mood stabilizers and the concomitant medications were $85.2,59.3$, and $44.4 \%$, respectively, these drugs might have influenced the improvement of psychopathology in the patients of the placebo arm. A recent network meta-analysis to examine the efficacy of antipsychotics (aripiprazole, haloperidol, molindone, olanzapine, paliperidone, quetiapine, risperidone, and ziprasidone) for the treatment of early-onset schizophrenia also reported that only three antipsychotics (molindone, olanzapine, and risperidone) were superior to placebo in total PANSS scores similar to this study. ${ }^{53}$

It is important to note that perospirone, which is approved for schizophrenia treatment only in Japan, was ranked as the most effective antipsychotic in the current meta-analysis for the improvement of the PANSS negative subscale scores. Although perospirone has not shown a greater reduction in negative symptoms than other SGAs (eg, aripiprazole, olanzapine, risperidone, and quetiapine) in previous open-label, 
Table 10 Network meta-analysis of triglyceride and potentially prolactin-related adverse events

\begin{tabular}{|c|c|c|c|c|c|}
\hline ARI & $\begin{array}{l}6.205 \\
(2.633,12.36)\end{array}$ & $\begin{array}{l}6.701 \\
(1.219,13.66)\end{array}$ & $\begin{array}{l}5.713 \\
(1.523,10.92)\end{array}$ & $\begin{array}{l}6.702 \\
(3.723,11.62)\end{array}$ & $\begin{array}{l}6.962 \\
(4.54, \text { II.52) }\end{array}$ \\
\hline \multirow[t]{3}{*}{$\begin{array}{l}-12.52 \\
(-41.95,16.89)\end{array}$} & BLO & $\begin{array}{l}0.4495 \\
(-5.386,5.857)\end{array}$ & $\begin{array}{l}-0.4843 \\
(-4.889,2.567)\end{array}$ & $\begin{array}{l}0.5221 \\
(-2.728,3.013)\end{array}$ & $\begin{array}{l}0.7685 \\
(-3.282,4.38 I)\end{array}$ \\
\hline & & CLO & $\begin{array}{l}-0.9754 \\
(-6.95,4.488)\end{array}$ & $\begin{array}{l}0.03455 \\
(-4.88,4.876)\end{array}$ & $\begin{array}{l}0.3176 \\
(-5.201,5.943)\end{array}$ \\
\hline & & & Cloca & $\begin{array}{l}0.9877 \\
(-1.721,4.419)\end{array}$ & $\begin{array}{l}1.275 \\
(-2,5.424)\end{array}$ \\
\hline $\begin{array}{l}-12.54 \\
(-35.58,11.68)\end{array}$ & $\begin{array}{l}-0.08063 \\
(-22.13,23.56)\end{array}$ & & & HAL & $\begin{array}{l}0.2779 \\
(-2.36,3.083)\end{array}$ \\
\hline $\begin{array}{l}-0.05474 \\
(-27.86,27.79)\end{array}$ & $\begin{array}{l}12.44 \\
(-28.04,52.84)\end{array}$ & & & $\begin{array}{l}12.57 \\
(-24.6,48.29)\end{array}$ & MOS \\
\hline $\begin{array}{l}-28.26 \\
(-58.08,2.637)\end{array}$ & $\begin{array}{l}-15.75 \\
(-45.76,15.66)\end{array}$ & & & $\begin{array}{l}-15.8 \\
(-41.48,10.36)\end{array}$ & $\begin{array}{l}-28.26 \\
(-68.12,13.72)\end{array}$ \\
\hline $\begin{array}{l}-38.06 \\
(-80.97,5.854)\end{array}$ & $\begin{array}{l}-25.72 \\
(-68.54,18.96)\end{array}$ & & & $\begin{array}{l}-25.61 \\
(-65.86,15.22)\end{array}$ & $\begin{array}{l}-38.2 \\
(-88.54,14.19)\end{array}$ \\
\hline $\begin{array}{l}-18.77 \\
(-60.87,24.8)\end{array}$ & $\begin{array}{l}-6.286 \\
(-48.64,37.72)\end{array}$ & & & $\begin{array}{l}-6.449 \\
(-45.81,33.91)\end{array}$ & $\begin{array}{l}-18.94 \\
(-68.55,33.11)\end{array}$ \\
\hline $\begin{array}{l}-8.031 \\
(-47.02,31.06)\end{array}$ & $\begin{array}{l}4.501 \\
(-37.37,47.12)\end{array}$ & & & $\begin{array}{l}4.484 \\
(-36.43,45.26)\end{array}$ & $\begin{array}{l}-7.998 \\
(-55.6,39.93)\end{array}$ \\
\hline $\begin{array}{l}-21.4 \\
(-50.06,6.405)\end{array}$ & $\begin{array}{l}-8.78 \\
(-32.16,13.07)\end{array}$ & & & $\begin{array}{l}-8.811 \\
(-35.99,15.86)\end{array}$ & $\begin{array}{l}-21.32 \\
(-61.83,17.59)\end{array}$ \\
\hline
\end{tabular}

Notes: Comparisons should be read from left to right. Mean differences (triglyceride) $<0$ favor the row-defining treatment. To obtain mean differences for comparisons in the opposing direction, negative values should be converted into positive values and vice versa. Log odds ratios (potentially prolactin-related adverse events) $<0$ favor the row-defining treatment. To obtain log odds ratio for comparisons in the opposing direction, negative values should be converted into positive values and vice versa. Significant

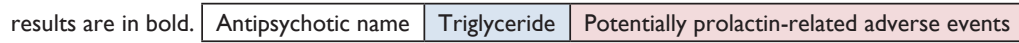

Abbreviations: ARI, aripiprazole; BLO, blonanserin; CLO, clozapine; Cloca, clocapramine; HAL, haloperidol; MOS, mosapramine; OLA, olanzapine; PAL, paliperidone; PBO, placebo; PER, perospirone; QUE, quetiapine; RIS, risperidone.

randomized trials, ${ }^{54,55}$ one double-blinded, randomized trial ${ }^{51}$ found that perospirone significantly reduces negative symptoms to a greater extent than haloperidol.

Clinical guidelines recommend that antipsychotics with a lower risk of adverse events should be selected for schizophrenia treatment. ${ }^{56-58}$ While negative impacts of adverse events on patients in the short term might be small or negligible, these long-standing adverse events can induce severe adverse consequences (eg, metabolic syndrome and diabetes), which are important risk factors for cardiovascular and cerebrovascular diseases. ${ }^{59}$ The current meta-analysis found that there were significant differences in individual adverse events between antipsychotics, similar to the findings of a previous meta-analysis. ${ }^{1}$

It was notable that blonanserin, which has been approved for schizophrenia treatment only in Japan and South Korea, was the first-ranked antipsychotic in the current network meta-analysis for the lowest risk of weight change. This is consistent with the result in previous meta-analyses, in which blonanserin exhibited a lower risk of weight gain than did risperidone. ${ }^{60,61}$
For response rate, a significant inconsistency between the direct and indirect estimates was detected between risperidone and haloperidol. There was only one study in this comparison. An inconsistency was also detected only regarding olanzapine versus quetiapine with respect to all-cause discontinuation. Risperidone, quetiapine, and olanzapine treatment groups differed from other antipsychotic treatment groups in that they included rater-blinded studies. ${ }^{41,42}$ Moreover, these studies which only included emergency cases might be a confounding factor to the results because unspecific effects such like rapid tranquilization might play a major role and specific antipsychotic effects may be overestimated. ${ }^{41,42}$ However, no clear reasons were found for these inconsistencies with respect to the outcomes.

The limitations of the present study need to be addressed. 1) The sample size was relatively limited. Additional efficacy and safety data are required to obtain a conclusive understanding. Specifically, only the comparison between olanzapine and risperidone included plural studies. Therefore, a sensitivity analysis was not performed when considerable heterogeneity was detected. Moreover, the generalization of 
Table 10 (Continued)

\begin{tabular}{|c|c|c|c|c|c|}
\hline $\begin{array}{l}-2.637 \\
(-213.3,70.31)\end{array}$ & $\begin{array}{l}37.9 \\
(-167.8,119.6)\end{array}$ & $\begin{array}{l}38.09 \\
(-167.9,119.4)\end{array}$ & $\begin{array}{l}7.234 \\
(3.668,12.63)\end{array}$ & $\begin{array}{l}-76.69 \\
(-186.5,1.365)\end{array}$ & $\begin{array}{l}7.893 \\
(4.404,14.48)\end{array}$ \\
\hline $\begin{array}{l}-8.777 \\
(-219.4,63.73)\end{array}$ & $\begin{array}{l}31.76 \\
(-174.3,113.9)\end{array}$ & $\begin{array}{l}31.94 \\
(-174.3,113.7)\end{array}$ & $\begin{array}{l}1.043 \\
(-3.384,4.715)\end{array}$ & $\begin{array}{l}-83.08 \\
(-193.2,-5.19)\end{array}$ & $\begin{array}{l}1.644 \\
(-0.815,4.906)\end{array}$ \\
\hline $\begin{array}{l}-9.468 \\
(-219,63.62)\end{array}$ & $\begin{array}{l}31.19 \\
(-174.6,112.8)\end{array}$ & $\begin{array}{l}31.32 \\
(-174.9,112.7)\end{array}$ & $\begin{array}{l}0.5645 \\
(-5.113,6.153)\end{array}$ & $\begin{array}{l}-83.29 \\
(-193.5,-5.259)\end{array}$ & $\begin{array}{l}1.238 \\
(-4.069,7.422)\end{array}$ \\
\hline $\begin{array}{l}-8.185 \\
(-218.5,64.65)\end{array}$ & $\begin{array}{l}32.51 \\
(-173.4,114.5)\end{array}$ & $\begin{array}{l}32.68 \\
(-173.4,114.2)\end{array}$ & $\begin{array}{l}1.514 \\
(-2.228,5.911)\end{array}$ & $\begin{array}{l}-82.42 \\
(-192.4,-4.319)\end{array}$ & $\begin{array}{l}2.165 \\
(-0.3132,6.544)\end{array}$ \\
\hline $\begin{array}{l}-9.173 \\
(-219.7,63.26)\end{array}$ & $\begin{array}{l}31.3 \\
(-174.4,113.3)\end{array}$ & $\begin{array}{l}31.48 \\
(-174.5,113.1)\end{array}$ & $\begin{array}{l}0.5247 \\
(-2.412,3.416)\end{array}$ & $\begin{array}{l}-83.54 \\
(-193.6,-5.646)\end{array}$ & $\begin{array}{l}1.139 \\
(-1.171,4.94)\end{array}$ \\
\hline $\begin{array}{l}-9.678 \\
(-220.4,62.96)\end{array}$ & $\begin{array}{l}30.95 \\
(-175.1,112.7)\end{array}$ & $\begin{array}{l}31.13 \\
(-175.2,112.6)\end{array}$ & $\begin{array}{l}0.2468 \\
(-3.04,3.338)\end{array}$ & $\begin{array}{l}-83.91 \\
(-193.7,-5.701)\end{array}$ & $\begin{array}{l}0.9053 \\
(-2.445,5.342)\end{array}$ \\
\hline OLA & $\begin{array}{l}37.16 \\
(-0.1568,94.1)\end{array}$ & $\begin{array}{l}36.99 \\
(-0.06605,93.97)\end{array}$ & $\begin{array}{l}9.742 \\
(-62.75,220.3)\end{array}$ & $\begin{array}{l}-67.45 \\
(-200.3,159.9)\end{array}$ & $\begin{array}{l}10.54 \\
(-61.91,221.3)\end{array}$ \\
\hline $\begin{array}{l}-9.871 \\
(-41.16,21.19)\end{array}$ & PAL & $\begin{array}{l}-0.0158 \\
(-4.969,4.946)\end{array}$ & $\begin{array}{l}-30.73 \\
(-112.8,175)\end{array}$ & $\begin{array}{l}-110.5 \\
(-251.9,125.9)\end{array}$ & $\begin{array}{l}-30.01 \\
(-112.2,176.1)\end{array}$ \\
\hline \multirow[t]{2}{*}{$\begin{array}{l}9.453 \\
(-20.88,39.65)\end{array}$} & $\begin{array}{l}19.28 \\
(-7.375,46.05)\end{array}$ & PBO & $\begin{array}{l}-30.87 \\
(-112.6,175.2)\end{array}$ & $\begin{array}{l}-110.4 \\
(-252.4,126)\end{array}$ & $\begin{array}{l}-30.21 \\
(-112,176.2)\end{array}$ \\
\hline & & & PER & $\begin{array}{l}-84.11 \\
(-194.1,-6.123)\end{array}$ & $\begin{array}{l}0.6118 \\
(-2.802,5.538)\end{array}$ \\
\hline $\begin{array}{l}20.18 \\
(-22.12,62.24)\end{array}$ & $\begin{array}{l}30.17 \\
(-22.8,82.31)\end{array}$ & $\begin{array}{l}10.79 \\
(-41.59,62.27)\end{array}$ & & QUE & $\begin{array}{l}84.81 \\
(6.893,194.9)\end{array}$ \\
\hline $\begin{array}{l}6.877 \\
(-23.26,35.2)\end{array}$ & $\begin{array}{l}16.81 \\
(-27.17,58.49)\end{array}$ & $\begin{array}{l}-2.543 \\
(-46.01,38.26)\end{array}$ & & $\begin{array}{l}-13.4 \\
(-53.36,25.57)\end{array}$ & RIS \\
\hline
\end{tabular}

results was limited because of the inclusion of studies treating Japanese patients only. 2) Although all studies included in this meta-analysis had a substantial dropout rate, $56 \%$ of the studies used FAS for the efficacy and safety analyses (the remaining 44\% studies used PPP). 3) Most (95\%) of the studies were sponsored by the pharmaceutical industry. Leucht et $\mathrm{al}^{62,63}$ showed that the results of the industrysponsored studies occasionally differed from those that were not industry-sponsored (sponsorship/publication bias). 4) It should also be addressed that the study did not include a meta-analysis using scores of extrapyramidal side effect rating scales and the use of antiparkinson drugs. RummelKluge et $\mathrm{al}^{64}$ reported that there were differences in the results between the severity of extrapyramidal side effect rating scales and the use of antiparkinson drugs. 5) Corrections for multiple comparisons (eg, using Bonferroni's correction) were not made because the application of a more stringent alpha level for secondary outcomes would have been overly conservative in this small sample. 6) There were some patients who also used additional antipsychotics (mean 9.1\%) and/or benzodiazepines (mean 25.8\%) at least once during the study for the majority of the studies included in the metaanalysis. 7) All trials included in this meta-analysis were of short duration ( $\leq 12$ weeks). Evidence from short duration studies may not be an appropriate basis of decision making concerning the safety of antipsychotics because adverse effects which occur right in the beginning (eg, akathisia) might be overvalued against long-term effects (eg, metabolic syndrome in several years). For example, this may influence the main outcome "all-cause discontinuation" in favor of olanzapine. Despite all of these limitations, the current results are coherent with those of the previous study of a completely different population with a larger sample size.

\section{Conclusion}

The current results suggest that olanzapine and paliperidone demonstrate efficacy and acceptability for short-term treatment in Japanese patients with schizophrenia. In agreement with the previous study, the safety outcomes exhibited diverse rankings for the antipsychotics, suggesting that clinical choices can be made according to the potential risks of adverse events in each patient. 


\section{Acknowledgments}

The authors thank Dr Kotaro Hatta, Otsuka Pharmaceutical Co Ltd, Eli Lilly and Company, Janssen Pharmaceutical KK, Astellas Pharma Inc., AstraZeneca plc, and Dainippon Sumitomo Pharma for providing information necessary for the study. KAKENHI (Grant-in-Aid for Young Scientists [B], ID: 26860949) was received for this study.

\section{Author contributions}

Drs Kishi and Ikuta had full access to all of the data in the study and bear responsibility for the integrity of the data and the accuracy of the data analysis. Kishi and Iwata were involved in the conceptualization and design of the study. Analysis and interpretation of data and statistical analysis were performed by Kishi, Ikuta, Matsunaga, Matsuda, and Oya. Data acquisition was carried out by Kishi, Matsunaga, Matsuda, and Oya and all authors were involved in drafting the manuscript. The study was supervised by Iwata. All authors contributed toward data analysis, drafting and critically revising the paper and agree to be accountable for all aspects of the work.

\section{Disclosure}

Dr Kishi has received speaker's honoraria from Daiichi Sankyo, Dainippon Sumitomo, Eisai, Eli Lilly, Janssen, Yoshitomi, Otsuka, Meiji, MSD, Tanabe-Mitsubishi, and Pfizer. Dr Ikuta has received speaker's honoraria from Daiichi Sankyo, Dainippon Sumitomo, and Eli Lilly Japan. Dr Matsunaga has received speaker's honoraria from Daiichi Sankyo, Dainippon Sumitomo, Eisai, Janssen, Meiji, Novartis, Otsuka, Takeda, and Tanabe-Mitsubishi and had a Fujita Health University School of Medicine research grant and Grant-in-Aid for Young Scientists (B). Dr Matsuda has received speaker's honoraria from Dainippon Sumitomo Eli Lilly and Otsuka and had a Fujita Health University School of Medicine research grant and Grant-in-Aid for Young Scientists (B). Dr Oya has received honoraria from Eli Lilly, Janssen, Otsuka, and Meiji. Dr Iwata has received speaker's honoraria from Astellas, Dainippon Sumitomo, Eli Lilly, GlaxoSmithKline, Janssen, Yoshitomi, Otsuka, Meiji, Shionogi, Novartis, and Pfizer and had research grants from Meiji, GlaxoSmithKline, and Otsuka.

\section{References}

1. Leucht S, Cipriani A, Spineli L, et al. Comparative efficacy and tolerability of 15 antipsychotic drugs in schizophrenia: a multiple-treatments meta-analysis. Lancet. 2013;382(9896):951-962.

2. Mattila T, Wohlfarth T, Koeter M, et al. Geographic variation in efficacy of atypical antipsychotics for the acute treatment of schizophrenia - an individual patient data meta-analysis. Eur Neuropsychopharmacol. 2014;24(7):1066-1077.
3. Chen YF, Wang SJ, Khin NA, Hung HM, Laughren TP. Trial design issues and treatment effect modeling in multi-regional schizophrenia trials. Pharm Stat. 2010;9(3):217-229.

4. Khin NA, Chen YF, Yang Y, Yang P, Laughren TP. Exploratory analyses of efficacy data from schizophrenia trials in support of new drug applications submitted to the US Food and Drug Administration. J Clin Psychiatry. 2012;73(6):856-864

5. Stauffer VL, Sniadecki JL, Piezer KW, et al. Impact of race on efficacy and safety during treatment with olanzapine in schizophrenia, schizophreniform or schizoaffective disorder. BMC Psychiatry. 2010;10:89.

6. Danaei G, Finucane MM, Lu Y, et al. National, regional, and global trends in fasting plasma glucose and diabetes prevalence since 1980: systematic analysis of health examination surveys and epidemiological studies with 370 country-years and 2.7 million participants. Lancet. 2011;378(9785):31-40.

7. Yoon KH, Lee JH, Kim JW, et al. Epidemic obesity and type 2 diabetes in Asia. Lancet. 2006;368(9548):1681-1688.

8. Sone H, Ito H, Ohashi Y, Akanuma Y, Yamada N; Japan Diabetes Complication Study Group. Obesity and type 2 diabetes in Japanese patients. Lancet. 2003;361(9351):85.

9. Slomko H, Heo HJ, Einstein FH. Minireview: epigenetics of obesity and diabetes in humans. Endocrinology. 2012;153(3):1025-1030.

10. Choi JH, Yoo HW. Control of puberty: genetics, endocrinology, and environment. Curr Opin Endocrinol Diabetes Obes. 2013;20(1):62-68.

11. Zandi PP, Judy JT. The promise and reality of pharmacogenetics in psychiatry. Clin Lab Med. 2010;30(4):931-974.

12. Reynolds GP. The pharmacogenetics of symptom response to antipsychotic drugs. Psychiatry Investig. 2012;9(1):1-7.

13. Mulligan MJ, Vasu R, Grossi CE, et al. Neoplastic meningitis with eosinophilic pleocytosis in Hodgkin's disease: a case with cerebellar dysfunction and a review of the literature. Am J Med Sci. 1988; 296(5):322-326.

14. The International HapMap Project [homepage on the Internet]. 2012 [updated June 16, 2016]. Available from: https://www.ncbi.nlm.nih. gov/variation/news/NCBI_retiring_HapMap/. Accessed March 22, 2017.

15. Malhotra AK, Correll CU, Chowdhury NI, et al. Association between common variants near the melanocortin 4 receptor gene and severe antipsychotic drug-induced weight gain. Arch Gen Psychiatry. 2012;69(9): 904-912.

16. Munro J, O’Sullivan D, Andrews C, Arana A, Mortimer A, Kerwin R. Active monitoring of 12,760 clozapine recipients in the UK and Ireland. Beyond pharmacovigilance. Br J Psychiatry. 1999;175:576-580.

17. Saito T, Ikeda M, Mushiroda T, et al. Pharmacogenomic study of clozapine-induced agranulocytosis/granulocytopenia in a Japanese population. Biol Psychiatry. 2016;80(8):636-642.

18. The International HapMap Project [homepage on the Internet]. 2012 [updated June 16, 2016]. Available from: https://www.ncbi.nlm.nih. gov/variation/news/NCBI_retiring_HapMap/. Accessed March 29, 2017.

19. Yamaguchi-Kabata Y, Nakazono K, Takahashi A, et al. Japanese population structure, based on SNP genotypes from 7003 individuals compared to other ethnic groups: effects on population-based association studies. Am J Hum Genet. 2008;83(4):445-456.

20. Leucht S, Chaimani A, Cipriani AS, Davis JM, Furukawa TA, Salanti G. Network meta-analyses should be the highest level of evidence in treatment guidelines. Eur Arch Psychiatry Clin Neurosci. 2016;266(6): 477-480.

21. Moher D, Liberati A, Tetzlaff J, Altman DG; Prisma Group. Preferred reporting items for systematic reviews and meta-analyses: the PRISMA statement. BMJ. 2009;339:b2535.

22. PROSPERO [homepage on the Internet]. 2009 [updated May, 2016]. Available from: https://www.crd.york.ac.uk/PROSPERO/\#index.php. Accessed March 22, 2017.

23. PubMed [homepage on the Internet]. January 1996. Available from: https://www.ncbi.nlm.nih.gov/pubmed. Accessed March 22, 2017.

24. Databases of Cochrane Library [homepage on the Internet].updated April 2013. http://www.cochranelibrary.com/. Accessed March 22, 2017. 
25. The Japan Medical Abstracts Society [homepage on the Internet]. updated November 2014. http://demo.jamas.or.jp/. Accessed March 22, 2017.

26. Pharmaceuticals and Medical Devices Agency [homepage on the Internet]. updated April 2017. https://www.pmda.go.jp/english/index. html. Accessed March 22, 2017.

27. Kay SR, Fiszbein A, Opler LA. The positive and negative syndrome scale (PANSS) for schizophrenia. Schizophr Bull. 1987;13(2):261-276.

28. DerSimonian R, Laird N. Meta-analysis in clinical trials. Control Clin Trials. 1986;7(3):177-188.

29. van Valkenhoef G, Lu G, de Brock B, Hillege H, Ades AE, Welton NJ. Automating network meta-analysis. Res Synth Methods. 2012;3(4): 285-299.

30. Higgins J, Green S. Cochrane Handbook for Systematic Reviews of Interventions Version 5.1.0. The Cochrane Collaboration; 2011. Available from: www.cochrane-handbook.org. Accessed April 24, 2017.

31. Salanti G, Higgins JP, Ades AE, Ioannidis JP. Evaluation of networks of randomized trials. Stat Methods Med Res. 2008;17(3):279-301.

32. Brooks S, Gelman A. General methods for monitoring convergence of iterative simulations. J Comput Graph Stat. 1998;7:434-455.

33. Dias S, Welton NJ, Sutton AJ, Caldwell DM, Lu G, Ades AE. Evidence synthesis for decision making 4: inconsistency in networks of evidence based on randomized controlled trials. Med Decis Making. 2013;33(5):641-656.

34. Dias S, Welton NJ, Caldwell DM, Ades AE. Checking consistency in mixed treatment comparison meta-analysis. Stat Med. 2010;29(7-8): 932-944.

35. Higgins JP, Thompson SG, Deeks JJ, Altman DG. Measuring inconsistency in meta-analyses. BMJ. 2003;327(7414):557-560.

36. Kudo Y, Nishimura K, Saito M, Otsuki S, Inanaga K, Nakajima T. A double-blind comparison of Y-516 with haloperidol in schizophrenic patients. J Clin Exp Med. 1990;152:529-542.

37. Kudo Y, Nakajima T, Saito M, et al. Clinical evaluation of a serotonin-2 and dopamine-2 receptor antagonist (SDA), perospirone HCI on schizophrenia - a comparative double-blind study with mosapramine HCI. Clin Eval. 1997;24:207-248.

38. Murasaki M. Clinical evaluation of blonanserin for schizophrenia: a double-blind trial comparing blonanserin with haloperidol. Jpn J Clin Psychopharmacol. 2007;10:2059Y2079.

39. Murasaki M, Koyama T, Fukushima H, et al. Clinical evaluation of quetiapine fumarate on schizophrenia: comparative double-blind study with haloperidol. Jpn J Clin Psychiatry. 2001;4(1):127-155.

40. Kudo Y, Nomura J, Igawa G, et al. Clinical evaluation of quetiapine fumarate for the treatment of schizophrenia: a double blind controlled study using mosapramine hydrochloride as a control. $J$ Clin Therapeutics Med. 2000;16(12):1807-1842.

41. Hatta K, Sato K, Hamakawa H, et al. Effectiveness of second-generation antipsychotics with acute-phase schizophrenia. Schizophr Res 2009;113(1):49-55.

42. Hatta K, Takebayashi H, Sudo Y, et al. The possibility that requiring high-dose olanzapine cannot be explained by pharmacokinetics in the treatment of acute-phase schizophrenia. Psychiatry Res. 2013; 210(2):396-401

43. Ishigooka J, Miura S, Koyama T, et al. Clinical evaluation of aripiprazole in schizophrenic patients: a comparative double-blind study with haloperidol. Jpn J Clin Psychopharmacol. 2006;9(2):295-329.

44. Ishigooka J, Inada T, Miura S. Olanzapine versus haloperidol in the treatment of patients with chronic schizophrenia: results of the Japan multicenter, double-blind olanzapine trial. Psychiatry Clin Neurosci. 2001;55(4):403-414.

45. Yagi G, Miura S, Tashiro I, et al. Comparison of clinical efficacy of clozapine and haloperidol in schizophrenia using double-blind technique. Clin Eval. 1974;2(2):169-206.

46. Miura S. Clinical evaluation of blonanserin for schizophrenia a randomized study comparing blonanserin with risperidone. Jpn J Clin Psychopharmacol. 2008;11:297-314.
47. Kurihara M, Ito N, Kato N, Kawakita Y, Kudo Y, Mori A. Clinical evaluation of clocapramine (Clofekton) in schizophrenia: a double blind comparison of clocapramine, haloperidol and perphenazine. Jpn J Clin Psychiatry. 1983;12(4):519-538.

48. Kudo Y, Nakajima T, Nishimura K, et al. Clinical evaluation of risperidone in schizophrenia: a double-blind comparative study of risperidone with clocapramine. Jpn J Clin Psychiatry. 1994;23:233-249.

49. Kato N, Takahashi R, Yagi G, Kazamatsuri H, Mori A, Murasaki M. A double-blind comparison of new iminodibenzyl compound Y-516 with clocapramine in the phamacotherapy of schizophrenia. Clin Eval. 1989;17:177-196.

50. Murasaki M, Yamashita I, Machiyama Y, et al. Efficacy of new antipsychotic, risperidone, on schizophrenia - a comparative double-blind study with haloperidol. Clin Eval. 1993;21:221-259.

51. Murasaki M, Koyama T, Yukiteru M, et al. Clinical evaluation of a new antipsychotic, perospirone HCI, on schizophrenia - a comparative double-blind study with haloperidol. Clin Eval. 1997;24:159-205.

52. Hirayasu Y, Tomioka M, Iizumi M, Kikushi H. A double-blind, placebocontrolled, comparative study of paliperidone extended-release (ER) tablets in patients with schizophrenia. Jpn J Clin Psychopharmacol. 2010;13:2077-2103.

53. Harvey RC, James AC, Shields GE. A systematic review and network meta-analysis to assess the relative efficacy of antipsychotics for the treatment of positive and negative symptoms in early-onset schizophrenia. CNS Drugs. 2016;30(1):27-39.

54. Takekita Y, Kato M, Wakeno M, et al. A 12-week randomized, openlabel study of perospirone versus aripiprazole in the treatment of Japanese schizophrenia patients. Prog Neuropsychopharmacol Biol Psychiatry. 2013;40:110-114.

55. Yamashita H, Mori K, Nagao M, Okamoto Y, Morinobu S, Yamawaki S. Effects of changing from typical to atypical antipsychotic drugs on subjective sleep quality in patients with schizophrenia in a Japanese population. J Clin Psychiatry. 2004;65(11):1525-1530.

56. Buchanan RW, Kreyenbuhl J, Kelly DL, et al. The 2009 schizophrenia PORT psychopharmacological treatment recommendations and summary statements. Schizophr Bull. 2010;36(1):71-93.

57. Hasan A, Falkai P, Wobrock T, et al. World Federation of Societies of Biological Psychiatry (WFSBP) guidelines for biological treatment of schizophrenia, part 1: update 2012 on the acute treatment of schizophrenia and the management of treatment resistance. World $J$ Biol Psychiatry. 2012;13(5):318-378.

58. NICE. Psychosis and Schizophrenia in Adults: Treatment and Management. London: National Institute for Health and Care Excellence (UK); 2014.

59. De Hert M, Detraux J, van Winkel R, Yu W, Correll CU. Metabolic and cardiovascular adverse effects associated with antipsychotic drugs. Nat Rev Endocrinol. 2012;8(2):114-126.

60. Kishi T, Matsuda Y, Nakamura H, Iwata N. Blonanserin for schizophrenia: systematic review and meta-analysis of double-blind, randomized, controlled trials. J Psychiatric Res. 2013;47(2):149-154.

61. Kishi T, Matsuda Y, Iwata N. Cardiometabolic risks of blonanserin and perospirone in the management of schizophrenia: a systematic review and meta-analysis of randomized controlled trials. PloS One. 2014;9(2):e88049.

62. Leucht S, Corves C, Arbter D, Engel RR, Li C, Davis JM. Secondgeneration versus first-generation antipsychotic drugs for schizophrenia: a meta-analysis. Lancet. 2009;373(9657):31-41.

63. Leucht S, Komossa K, Rummel-Kluge C, et al. A meta-analysis of head-to-head comparisons of second-generation antipsychotics in the treatment of schizophrenia. Am J Psychiatry. 2009;166(2):152-163.

64. Rummel-Kluge C, Komossa K, Schwarz S, et al. Second-generation antipsychotic drugs and extrapyramidal side effects: a systematic review and meta-analysis of head-to-head comparisons. Schizophr Bull. 2012;38(1):167-177. 


\section{Publish your work in this journal}

Neuropsychiatric Disease and Treatment is an international, peerreviewed journal of clinical therapeutics and pharmacology focusing on concise rapid reporting of clinical or pre-clinical studies on a range of neuropsychiatric and neurological disorders. This journal is indexed on PubMed Central, the 'PsycINFO' database and CAS,

and is the official journal of The International Neuropsychiatric Association (INA). The manuscript management system is completely online and includes a very quick and fair peer-review system, which is all easy to use. Visit http://www.dovepress.com/testimonials.php to read real quotes from published authors.

Submit your manuscript here: http://www.dovepress.com/neuropsychiatric-disease-and-treatment-journal 